ARTICLE

Received 25 Sep 2014 | Accepted 4 Aug 2015 | Published 30 Sep 2015 DOl: 10.1038/ncomms9271

\title{
PBX3 is targeted by multiple miRNAs and is essential for liver tumour-initiating cells
}

Haibo Han ${ }^{1}$, Yantao Du ${ }^{1}$ Wei Zhao ${ }^{1}$, Sheng $\mathrm{Li}^{1}$, Dongji Chen ${ }^{1}$, Jing Zhang ${ }^{2}$, Jiang Liu $^{2}$, Zhenhe Suo ${ }^{3}$, Xiuwu Bian ${ }^{4}$, Baocai Xing ${ }^{5} \&$ Zhiqian Zhang $^{1}$

Tumour-initiating cells (TICs) are advocated to constitute the sustaining force to maintain and renew fully established malignancy; however, the molecular mechanisms responsible for these properties are elusive. We previously demonstrated that voltage-gated calcium channel $\alpha 2 \delta 1$ subunit marks hepatocellular carcinoma (HCC) TICs. Here we confirm directly that $\alpha 2 \delta 1$ is a HCC TIC surface marker, and identify let-7c, miR-200b, miR-222 and miR-424 as suppressors of $\alpha 2 \delta 1+$ HCC TICs. Interestingly, all the four miRNAs synergistically target $P B X 3$, which is sufficient and necessary for the acquisition and maintenance of TIC properties. Moreover, PBX3 drives an essential transcriptional programme, activating the expression of genes critical for HCC TIC stemness including CACNA2D1, EPCAM, SOX2 and NOTCH3. In addition, the expression of CACNA2D1 and PBX3 mRNA is predictive of poor prognosis for HCC patients. Collectively, our study identifies an essential signalling pathway that controls the switch of HCC TIC phenotypes.

\footnotetext{
${ }^{1}$ Department of Cell Biology, Key Laboratory of Carcinogenesis and Translational Research (Ministry of Education/Beijing), Center for Molecular and Translational Medicine, Peking University Cancer Hospital and Institute, 52 Fucheng Road, Beijing 100142, China. ${ }^{2}$ Beijing Institute of Genomics, Chinese Academy of Sciences, Beijing 100101, China. ${ }^{3}$ Department of Pathology, Oslo University Hospital, Institute of Clinical Medicine, Faculty of Medicine, University of Oslo, Oslo 0379, Norway. ${ }^{4}$ Institute of Pathology and Southwest Cancer Center, Southwest Hospital, Third Military Medical University, Chongqing 400038, China. ${ }^{5}$ Department of Hepatobiliary Surgery I, Peking University Cancer Hospital and Institute, Beijing 100142, China. Correspondence and requests for materials should be addressed to Z.Z. (email: zlzqzhang@bjmu.edu.cn).
} 
A s a highly tumorigenic and drug-resistant subpopulation, tumour-initiating cells (TICs) or cancer stem cells (CSCs) own many stem cell-like properties such as self-renewal and differentiation ${ }^{1-3}$. TICs are able to generate the heterogeneous lineages of cancer cells that comprise the tumour, and hence have been advocated to constitute the sustaining force to maintain and renew fully established malignancy. These properties of TICs have therefore led to the proposal that TICs are responsible for tumour occurrence, metastasis and recurrence, and TIC-targeted therapeutic approaches may provide promising strategies to improve clinical cancer therapy ${ }^{4}$.

Although exactly how TICs emerge remains elusive, accumulating evidence demonstrates that TICs may be the oncogenic derivatives of normal-tissue stem or progenitor cells, or arise from acquisition of stem cell properties by more differentiated cells as a result of transformation-associated genetic or epigenetic reprogramming ${ }^{5-10}$. Furthermore, recent studies indicate that CSC phenotypes can reversibly turn on and off spontaneously or with the variation of their microenvironment niches ${ }^{11-14}$. Uncovering the molecular pathways involved in the control of TIC properties is critical for the understanding of TIC biology as well as for the development of novel therapies ${ }^{3}$. However, functional identification of such molecules at genome-wide scale is usually hindered by the limited availability and unstable properties of TICs.

MicroRNAs (miRNA), a large family of short noncoding RNAs that negatively regulate mRNA stability or translation through imperfect base pairing with the $3^{\prime}$-untranslated regions (UTRs) of target genes, have been functionally linked to stem cells ${ }^{15-18}$ and TICs or CSCs ${ }^{19}$. In fact, aberrant expression of miRNAs such as let-7, miR-34a, miR-181a, miRNA-130b in TICs has been found in a variety of tumour types. These aberrantly expressed miRNAs play essential roles in the regulation of TIC properties as either TIC suppressors or promoters ${ }^{20-24}$. Moreover, a series of critical signalling pathways involved in the regulation of TIC properties have been uncovered with the identification of the targets of these miRNAs ${ }^{19,25-28}$. Importantly, miRNAs are also demonstrated to be promising biomarkers and amenable therapeutic targets $5,19,24$.

On the basis of a pair of hepatocellular carcinoma (HCC) cell lines Hep-11 and Hep-12, which represent non-tumorigenic and TIC-enriched cell populations, respectively ${ }^{29}$, we identified a subpopulation of TICs expressing the isoform 5 of the voltagegated calcium channel $\alpha 2 \delta 1$ subunit (encoded by the gene CACNA2D1), which is vital for the activation of calcium signalling that controls the HCC TIC properties using an antibody, Mab1B50-1, generated against Hep-12 cells ${ }^{30,31}$. Here we first validate directly that $\alpha 2 \delta 1$ is indeed a surface marker for HCC TICs using a known $\alpha 2 \delta 1$ antibody and identify its prognostic role in HCC. Subsequently, we identify $4 \propto 2 \delta 1^{+}$TIC suppressor miRNAs (let-7c, miR-200b, miR-222 and miR-424) that synergistically target PBX3, which is essential for $\alpha 2 \delta 1^{+}$ HCC TIC properties through genome-wide miRNA profiling of Hep-11 and Hep-12 cells, followed by soft-agar formation functional screening. Our studies indicate that downregulation of the four miRNAs is responsible for the acquisition and subsequent maintenance of $\alpha 2 \delta 1^{+}$TIC phenotypes via common target PBX3, which serves as a transcriptional switch between TIC and non-TIC programming.

\section{Results}

Confirmation of $\boldsymbol{\alpha} \mathbf{2 \delta 1}$ as a HCC TIC marker. To directly confirm that $\alpha 2 \delta 1$ is a surface marker that marks HCC TICs, we purified both $\alpha 2 \delta 1^{+}$and $\alpha 2 \delta 1^{-}$cells using a known $\alpha 2 \delta 1$ antibody using fluorescence-activated cell sorting (FACS) from
HCC cell lines, including Huh7, HepG2, SMMC7721, as well as primary HCC tissues to assay their self-renewal and tumorigenic properties. As shown in Fig. 1a,b, purified $\alpha 2 \delta 1^{+}$cells formed spheres in serum-free medium at significantly higher rates than their negative counterparts. Furthermore, single cells obtained from these $\alpha 2 \delta 1^{+}$-dissociated spheres could be clonally expanded and serially propagated, demonstrating that these $\alpha 2 \delta 1^{+}$ cells possess in vitro self-renewal capability.

Next, we transplanted serially diluted $\alpha 2 \delta 1^{+}$and $\alpha 2 \delta 1^{-}$cells subcutaneously (s.c.) into nonobese diabetes/severe combined immunodeficient (NOD/SCID) mice to evaluate their tumorigenic potential. As few as 100 purified $\alpha 2 \delta 1^{+}$cells from HCC cell lines and primary tumours were able to initiate tumour formation in almost all of the transplanted mice in 8 weeks following transplantation, whereas the $\alpha 2 \delta 1^{-}$cells were not observed for any nodules formed within 12 weeks (Fig. 1c,d; Table 1; Supplementary Fig. 1). In addition, when $\alpha 2 \delta 1^{+}$and $\alpha 2 \delta^{-}$ cells sorted from these primary tumour xenografts were retransplanted into secondary mouse recipients, only $\alpha 2 \delta 1^{+}$ cells successfully resulted in tumour formation, indicating that tumorigenic $\alpha 2 \delta 1+$ cells have the in vivo self-renewal potential (Table 1; Supplementary Fig. 1). Histological analysis with the tumours formed by $\alpha 2 \delta 1^{+}$cells by haematoxylin and eosine (HE) staining showed that the tumour morphologies are indistinguishable from their parent cells (Fig. 1e).

These data, along with our previous results with Mab1B50-1 (ref. 30), demonstrate clearly that a TIC population with stem cell-like properties in HCC could be defined by a surface $\alpha 2 \delta 1$ phenotype.

Clinical significance of $\boldsymbol{\alpha} \mathbf{2} \boldsymbol{\delta} 1$ expression in HCC. Since TICs are usually rare in tumour tissues, it is hard to quantify the immunohistochemical staining results. We therefore performed quantitative reverse transcription-polymerase chain reaction (qRT-PCR) analysis in 89 paired HCC and adjacent normal tissues to test the clinical significance of $\alpha 2 \delta 1$. The $\alpha 2 \delta 1$ mRNA level in cancer tissues was remarkably higher than that of matched normal tissues (Fig. 1f). Further analysis of $\alpha 2 \delta 1$ mRNA expression in cancer tissues from 85 HCC patients with detailed follow-up information showed that high level of $\alpha 2 \delta 1$ mRNA was positively correlated not only with tumour size, but also with rapid recurrence and short overall survival post surgery (Supplementary Table 1). Kaplan-Meier survival curves showed that the patients with high level of $\alpha 2 \delta 1$ mRNA displayed both shorter tumour-free survival (Fig. $1 \mathrm{~g}$ ) and overall survival periods (Fig. 1h). Cox regression analysis also identified that high level of $\alpha 2 \delta 1 \mathrm{mRNA}$ is an independent risk factor of poor survival for HCC patients (relative risk $=2.66, P=0.005$, Supplementary Table 2), suggesting that the $\alpha 2 \delta 1$ mRNA level in HCC may serve as a prognostic factor.

Identification of miRNAs that suppress Hep-12 cell stemness. To find miRNAs that regulate the stemness of HCC TICs, we took advantage of the TIC-enriched Hep-12 and the nontumorigenic Hep-11 cell lines by genome-wide profiling of miRNA expression with miRNA microarray analysis, followed by functional screening with soft-agar assay (Fig. 2a).

Comparing with Hep-11 cells, 31 mature miRNAs including those that have been reported to have a tumour-suppressor role in other types of TICs such as let-7 family members, miR-200b and miR-34a, were found to be downregulated, and 46 mature miRNAs including miR-130b, which acted as an oncomir in $\mathrm{CD}_{133^{+}} \mathrm{HCC} \mathrm{TICs}^{21}$, were upregulated significantly in Hep-12 cells (Fig. 2b). 

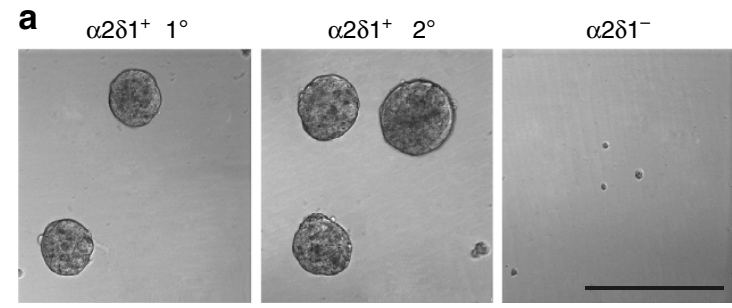

c

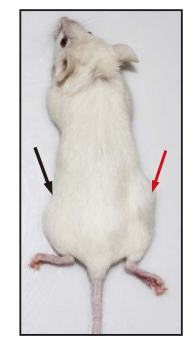

d

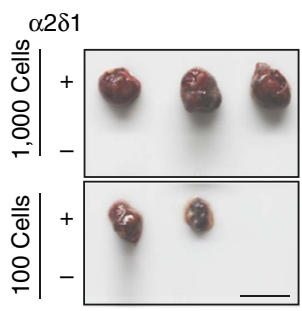

e

g

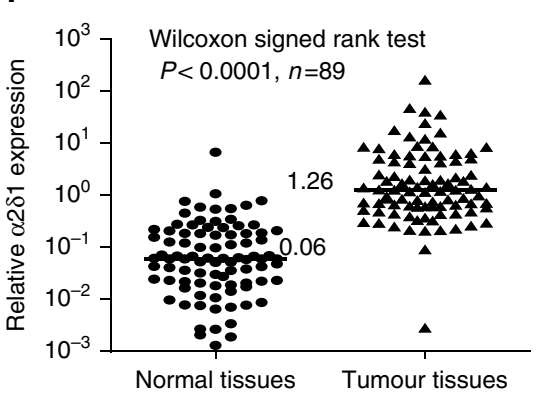

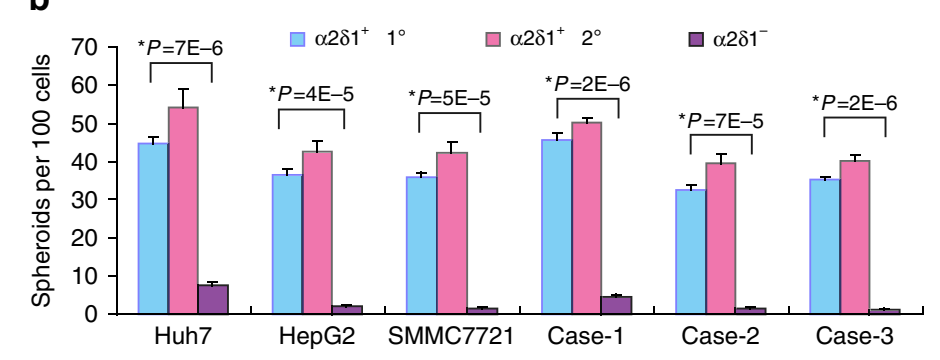

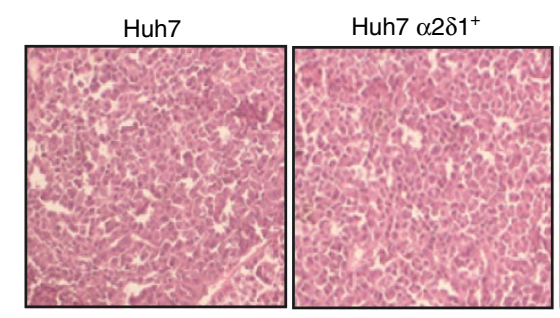

b

Case-2
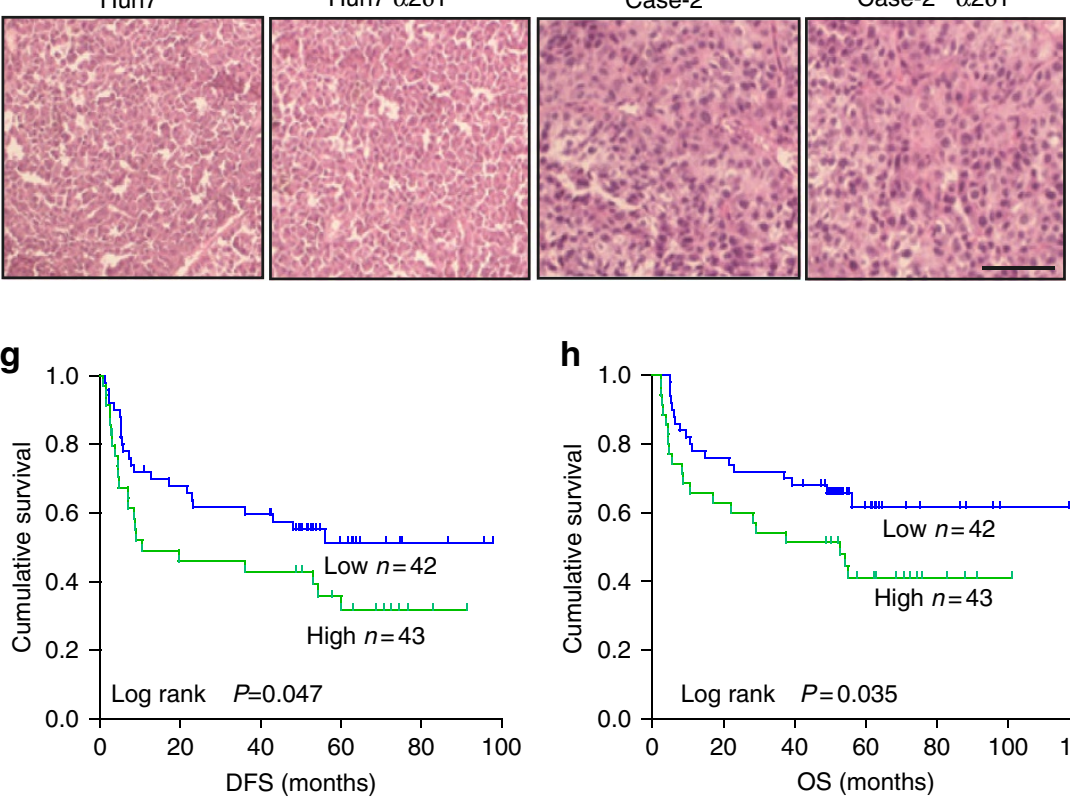

h

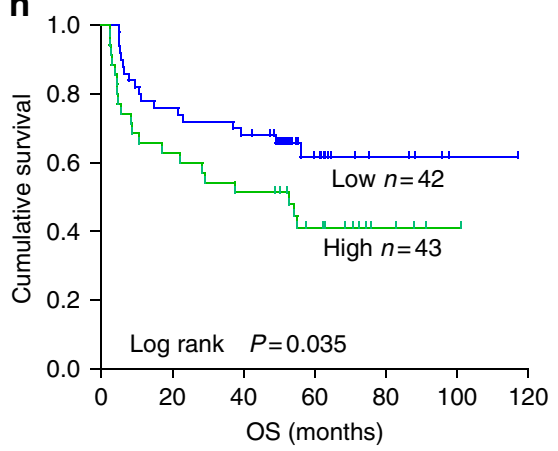

Figure 1 | The tumour-initiating properties of $\boldsymbol{\alpha 2} \mathbf{2} \mathbf{1}^{+} \mathbf{H C C}$ cells. (a) Phase contrast micrographs demonstrate that FACS-sorted $\alpha 2 \delta 1^{+}$Huh7 cells are able to form more primary $\left(1^{\circ}\right)$ and serially passaged $\left(2^{\circ}\right)$ spheroids as compared with $\alpha 2 \delta 1^{-}$counterparts. Scale bar, $250 \mu \mathrm{m}$. (b) Histograms show the primary $\left(1^{\circ}\right)$ and serially passaged $\left(2^{\circ}\right)$ spheroid formation efficiency of the $\alpha 2 \delta 1^{+}$cells as compared with $\alpha 2 \delta 1^{-}$cells sorted from indicated sources. Bars are the mean \pm s.d. of three independent experiments $(n=6)$. * Student's $t$-test. (c) Representative photograph showing tumour formation in NOD/SCID mice was restricted to the $\alpha 2 \delta 1^{+}$cell population (red arrow). No tumour growth was usually observed on injection of $\alpha 2 \delta 1^{-}$cells (black arrow) on the opposite flanks of the same animals. (d) Representative images showing the dissected tumours formed by sorted $\alpha 2 \delta 1^{+} \mathrm{Huh} 7 \mathrm{cells}$. A total of three mice per group were transplanted. Scale bar, $2 \mathrm{~cm}$. (e) The histology of tumours formed by $\alpha 2 \delta 1^{+}$cells purified from a primary HCC tissue (Case-2) and Huh7 cell line was compared with that of original patient tumour and parent Huh7 tumour, respectively, by HE staining. Scale bar, $100 \mu \mathrm{m}$. (f) qRT-PCR analysis of $\alpha 2 \delta 1$ mRNA levels in HCC tissues and paired normal tissues adjacent to tumours. Horizon lines indicate the median values of each group. (g,h) KaplanMeier curves for the disease-free survival (DFS) and overall survival (OS) of $85 \mathrm{HCC}$ patients were compared between groups with high and low levels of $\alpha 2 \delta 1$ mRNA in HCC tissues, which were divided according to the cutoff of 1.26 , the median value of $\alpha 2 \delta 1$ relative to GAPDH mRNA.

\section{Table 1 | Engraftment rates of $\alpha \mathbf{2 8 1 ^ { + }}$ and $\alpha \mathbf{2 \delta 1}$ subpopulations in NOD/SCID mice.}

\begin{tabular}{|c|c|c|c|c|c|}
\hline \multirow[t]{2}{*}{ Cells } & \multirow[t]{2}{*}{$\alpha 2 \delta 1$} & \multicolumn{2}{|c|}{ Primary transplantation } & \multicolumn{2}{|c|}{ Second transplantation } \\
\hline & & $10^{3}$ & $10^{2}$ & $10^{3}$ & $10^{2}$ \\
\hline \multirow[t]{2}{*}{ Huh7 } & + & $3 / 3$ & $2 / 3$ & ND & ND \\
\hline & - & $0 / 3$ & $0 / 3$ & ND & ND \\
\hline \multirow[t]{2}{*}{ HepG2 } & + & $3 / 3$ & $3 / 3$ & $3 / 3$ & $3 / 3$ \\
\hline & - & $0 / 3$ & $0 / 3$ & $0 / 3$ & $0 / 3$ \\
\hline \multirow[t]{2}{*}{ SMMC7721 } & + & $3 / 3$ & $3 / 3$ & $3 / 3$ & $2 / 3$ \\
\hline & - & $0 / 3$ & $0 / 3$ & $1 / 3$ & $0 / 3$ \\
\hline \multirow[t]{2}{*}{ Case-1 } & + & $3 / 3$ & $3 / 3$ & ND & ND \\
\hline & - & $0 / 3$ & $0 / 3$ & ND & ND \\
\hline \multirow[t]{2}{*}{ Case-2 } & + & $3 / 3$ & $2 / 3$ & $3 / 3$ & $3 / 3$ \\
\hline & - & $0 / 3$ & $0 / 3$ & $0 / 3$ & $0 / 3$ \\
\hline \multirow[t]{2}{*}{ Case-3 } & + & $3 / 3$ & $3 / 3$ & $3 / 3$ & $2 / 3$ \\
\hline & - & $0 / 3$ & $0 / 3$ & $0 / 3$ & $0 / 3$ \\
\hline
\end{tabular}

Each expression construct of those downregulated miRNAs was then transfected into Hep-12 cells, and the G418-resistant cell pools were assayed for their abilities to grow in soft agar. A total of nine miRNAs including let-7c, mir-126, mir-200b, mir-221, mir-222, mir-224, mir-31, mir-424 and mir-455 were able to inhibit the colony formation of Hep-12 cells significantly compared with the vector alone control $(P<0.05$, Fig. 2c). Tetracycline-inducible Hep-12 cells expressing each of the above nine miRNAs were further established by employing the TET-on lentiviral system (Fig. 2d), and $10^{4}$ cells per mouse were injected s.c. into NOD/SCID mice to evaluate the change of tumorigenic property after doxocycline (DOX) was administrated in drinking water. Interestingly, induced expression of these nine miRNAs could remarkably retard in vivo tumour growth of Hep-12 cells, while let-7c, miR-200b, miR-222 and miR-424 resulted in a complete inhibition of tumour formation (Fig. 2e). 
a

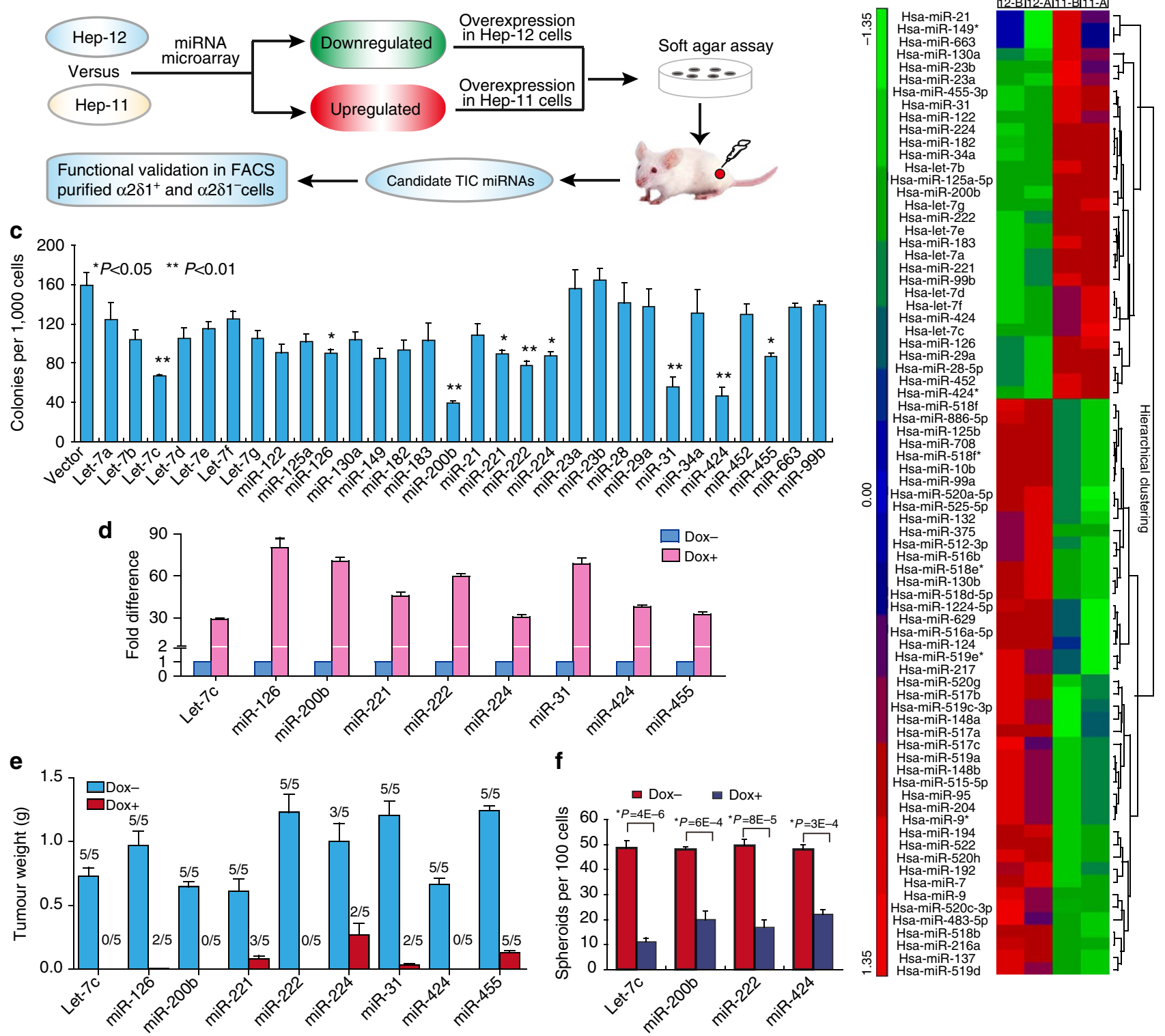

Figure 2 | Identification of candidate miRNAs that control $\mathbf{\alpha 2 \delta \mathbf { 1 } ^ { + }}$ HCC TICs. (a) Schematic overview of the strategy for identification of $\alpha 2 \delta 1^{+} \mathrm{HCC}$ TIC miRNAs based on the Hep-11 and Hep-12 cell lines. (b) Heat map of miRNA expression showing the miRNAs deregulated in the TIC-enriched Hep-12 cells versus the non-tumorigenic Hep-11 cells $(P<0.01)$. Clustering was performed with average linkage and uncentred correlation using the Partek Genomics Suite (Partek SG Pte Ltd, Singapore). (c) The effect of indicated miRNAs on the colony formation efficiency of Hep-12 cells in soft agar. Pools of Hep-12 cells stably transfected with each indicated pri-mRNA were plated into $0.3 \%$ agar at 1,000 cells per well $(n=6)$. Error bars represent s.d. ${ }^{*}$ Student's $t$-test. (d) qRT-PCR results demonstrate that the indicated miRNAs are induced significantly at $48 \mathrm{~h}$ after $2 \mu g \mathrm{ml}^{-1}$ DOX was added into the culture medium of Hep-12 cells, which were stably infected with tet-on pri-miRNA expression lentiviruses. Error bars represent s.d. (e). Tumour formation by Hep-12 cells stably infected with inducible pri-miRNA expression lentiviruses were assayed after transplanting $10^{4}$ cells in Matrigel subcutaneously. To induce the miRNA expression, mice in the DOX $(+)$ group were fed with water containing $2 \mathrm{mg} \mathrm{ml}^{-1}$ DOX. The numbers above each bar indicate the tumourformation rates that are expressed as tumours formed/sites transplanted. (f) The spheroid formation efficiency of Hep-12 cells after the expression of indicated miRNAs was induced with $2 \mu \mathrm{g} \mathrm{ml}^{-1} \mathrm{DOX}(n=6)$. Data are means \pm s.d. of three independent experiments. ${ }^{\star}$ Student's $t$-test.

The four most effective miRNAs, let-7c, miR-200b, miR-222 and miR-424, were further tested for their effects on the selfrenewal ability of Hep-12 cells by spheroid formation assay. The spheroid formation efficiency decreased significantly when the expression of the four miRNAs was induced individually by DOX $(P \leq 0.001$, Fig. $2 f$ ).

Therefore, we have identified four miRNAs, let-7c, miR-200b, miR-222 and miR-424, which are downregulated in Hep-12 cells and are able to suppress the self-renewal and tumorigenic properties of Hep-12 cells.
The suppression roles of the four miRNAs on HCC TICs. To validate that these four miRNAs are indeed involved in the regulation of $\alpha 2 \delta 1^{+}$HCC TICs in general, we first detected their expression in FACS-purified $\alpha 2 \delta 1^{+}$and $\alpha 2 \delta 1^{-}$subsets using qRT-PCR. As expected, the four miRNAs were found to be remarkably downregulated in $\alpha 2 \delta 1^{+}$subsets from a variety of HCC cell lines and primary tissues comparing with their $\alpha 2 \delta 1^{-}$ counterparts (Fig. 3a). The downregulation of the four miRNAs in $\alpha 2 \delta 1^{+}$TICs was further confirmed by miRNA fluorescence in situ hybridization (Supplementary Fig. 2). 
a

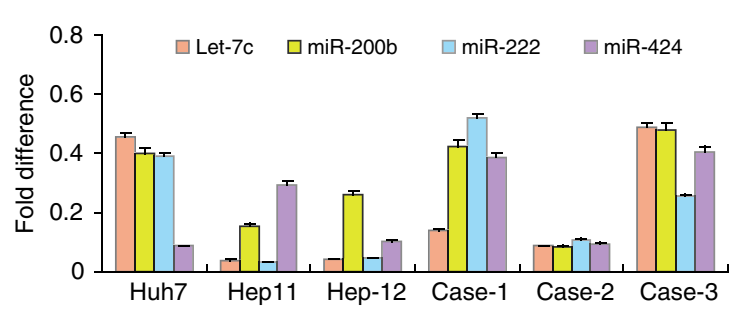

C

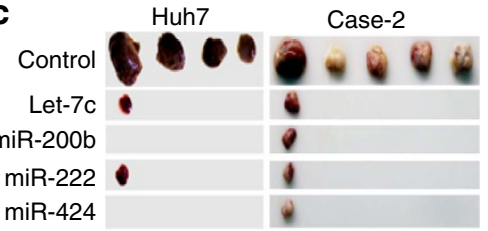

d

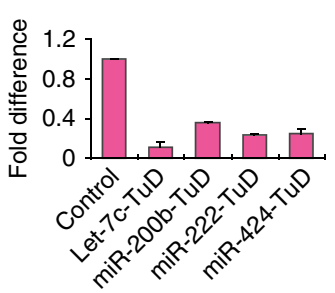

$\mathbf{f}$

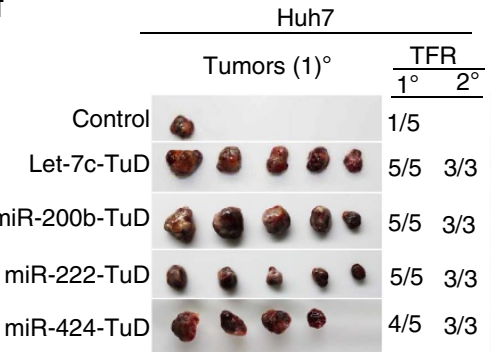

e

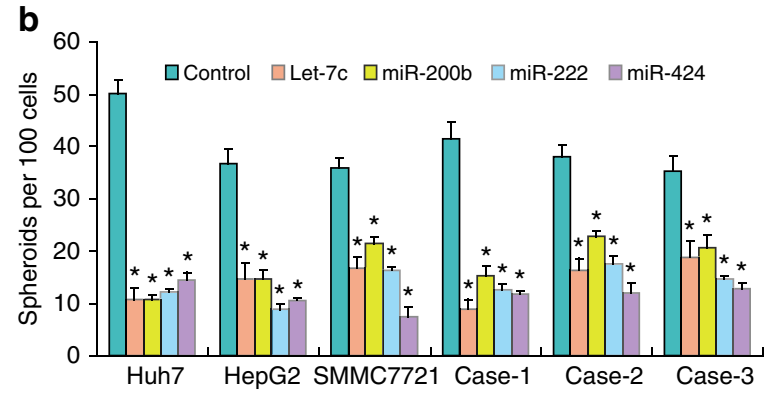

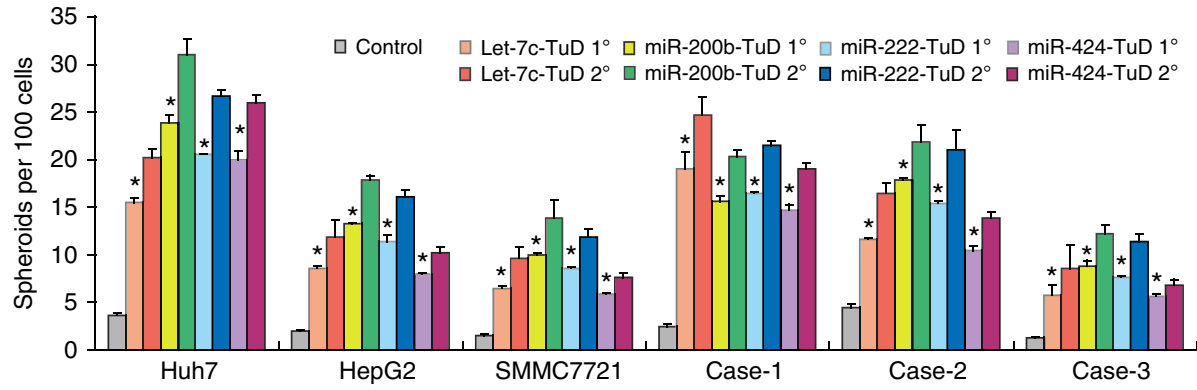

h
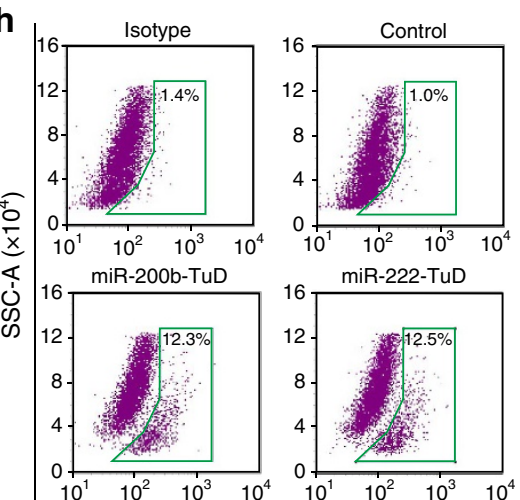

Let-7c-TuD
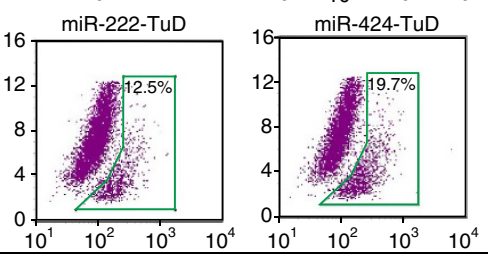

g
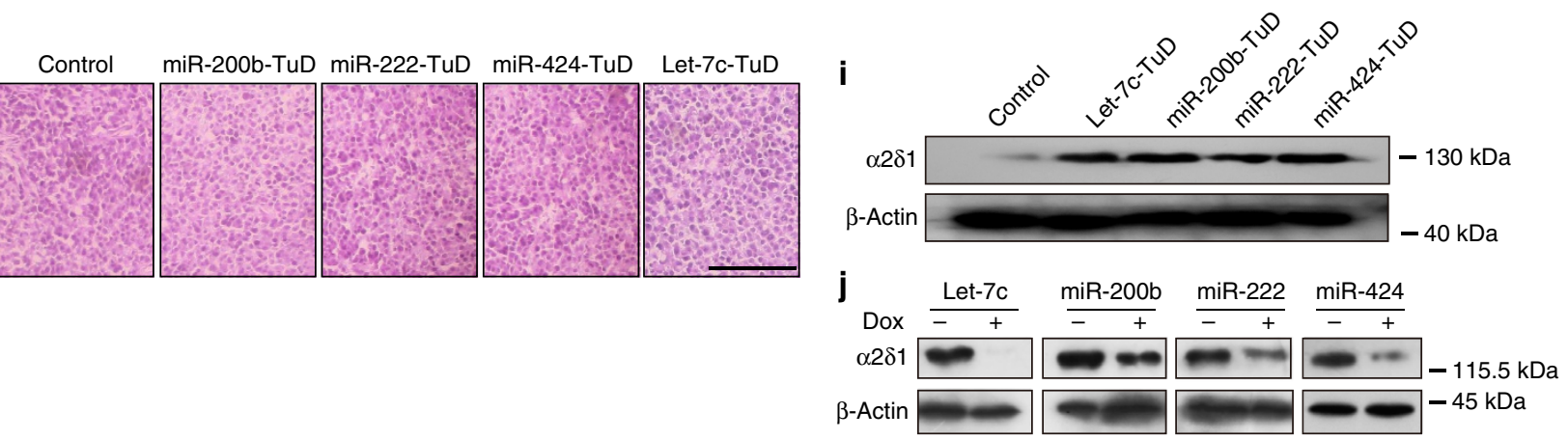

Figure 3 | The effects of let-7c, miR-200b, miR-222 and miR-424 on the self-renewal and tumorigenicity of HCC TICs. (a) The expression of indicated miRNAs in $\alpha 2 \delta 1^{+}$and $\alpha 2 \delta^{-}$cells purified from indicated sources were detected using qRT-PCR. The data presented as fold difference of $\alpha 2 \delta 1^{+}$cells over $\alpha 2 \delta^{-}$counterparts that was defined as 1 (omitted in the histogram). Error bars indicate s.d. (b,c) FACS-sorted $\alpha 2 \delta 1^{+}$fractions were incubated with indicated lentiviruses for $2 \mathrm{~h}$ and were plated in 96-well plates at 100 cells per well $(n=6)$ to assay their spheroid formation efficiency (b), or were transplanted into NOD/SCID mice $(n=5)$ at 1,000 cells per site to test their tumorigenicity (c). Scale bar, $2 \mathrm{~cm}$. Data in (b) are means \pm s.d. of three independent experiments. *Student's $t$-test, $P<0.01$ versus respective controls. (d) The fold difference $\left(2^{-\Delta \Delta C t}\right)$ of indicated miRNAs in Huh7 $\alpha 2 \delta 1^{-}$cells following TuD RNA interference of the indicated miRNAs over the control (infected with empty virus), which was set up as 1. Error bars indicate s.d. of three experiments. (e,f) FACS-sorted $\alpha 2 \delta 1^{-}$cells were infected with lentiviruses harbouring TuD RNA expression constructs against indicated miRNAs and were subsequently plated into 96-well plates to assay their primary spheroid formation efficiency $\left(1^{\circ}\right)$ and subsequent expansion ability $\left(2^{\circ}, \mathbf{e} ; n=6\right)$, or serially transplanted s.c. into NOD/SCID mice at 1,000 cells per site $(\mathbf{f} ; n=5)$. Scale bar, $2 \mathrm{~cm}$. TFR, tumour-formation rate that is expressed as tumours formed/sites transplanted. Data in (e) are means \pm s.d. of three independent experiments. ${ }^{*}$ Student's $t$-test, $P<0.01$ versus respective controls. (g) HE staining demonstrating the histology of the tumours formed in $\mathbf{f}$. Scale bar, $150 \mu \mathrm{m}$. (h) The frequencies of $\alpha 2 \delta 1^{+}$cells were measured by flow cytometry in Huh7 cells after indicated miRNAs were knocked down by infection with Tud RNA lentiviruses. The data are representative of three experiments. (i,j) Western blot analysis of $\alpha 2 \delta 1$ expression in Huh7 cells after indicated miRNAs were knocked down (i) and in Hep-12 cells after induced expression of indicated miRNAs (j). 
We then overexpressed the four miRNAs individually in FACS-purified $\alpha 2 \delta 1^{+}$TICs from HCC cell lines and primary tissues to test their effects on the self-renewal capability. Forced expression any of the four miRNAs resulted in remarkable inhibition of spheroid formation of $\alpha 2 \delta 1^{+}$cells, indicating that these miRNAs could suppress the in vitro self-renewal ability of $\alpha 2 \delta 1^{+}$HCC TICs (Fig. 3b).

Next, 1,000 sorted $\alpha 2 \delta 1^{+}$cells from Huh7 cell line and a patient biopsy, which were incubated with lentiviruses harbouring each of the four miRNAs, were transplanted s.c. into NOD/SCID mice to test their tumorigenicity. Comparing with the control lentivirus-infected cells, the tumorigenicity of $\alpha 2 \delta 1^{+}$TICs was significantly suppressed with any of the four miRNAs overexpressed (Fig. 3c).

Hence, let-7c, miR-200b, miR-222 and miR-424 could indeed suppress the self-renewal and tumorigenic properties of $\alpha 2 \delta 1^{+}$ TICs.

The knockdown effects of the four miRNAs. We furthered our study to test whether the downregulation of the four miRNAs individually is sufficient to convert $\alpha 2 \delta 1^{-}$cells into TIC-like cells by knockdown of the four miRNAs individually using the tough decoy $(\mathrm{TuD}) \mathrm{RNA}$ method $^{32}$. The expression of let-7c, miR-200b, miR-222 and miR-424 was inhibited $\sim 89.3 \%, 64.3 \%, 76.6 \%$ and $75.0 \%$, respectively, in $\alpha 2 \delta 1^{-}$cells purified from Huh7 cells by TuD RNAs against each miRNA compared with control (Fig. 3d).

Next, we performed spheroid formation assay to measure whether the $\alpha 2 \delta 1^{-}$cells could acquire in vitro self-renewal ability following these miRNAs' knockdown. The spheroid formation efficiency of $\alpha 2 \delta 1^{-}$cells was remarkably promoted following knockdown of the four miRNAs individually. Furthermore, single cells obtained from these dissociated spheres could be clonally expanded in subsequent serial propagations with increased efficiency, demonstrating that these $\alpha 2 \delta 1^{-}$cells acquired in vitro self-renewal capability after individual knockdown of the four miRNAs. Similar results were obtained when the four miRNAs were interfered in $\alpha 2 \delta 1^{-}$cells purified from more HCC cell lines and primary tissues (Fig. 3e).

We then transplanted $10^{3} \alpha 2 \delta 1^{-}$cells purified from the Huh7 cell line and a primary HCC tissue into NOD/SCID mice to evaluate their tumorigenicity following individual knockdown of the four miRNAs. Newly acquired or enhanced tumorigenicity was observed for $\alpha 2 \delta 1^{-}$cells after knockdown any of these four miRNAs. Furthermore, these primary xenografted tumours could also self-renew, as demonstrated by their ability to form new tumours when serially transplanted in secondary mouse recipients (Fig. 3f). HE staining confirmed that the tumours formed were of HCC histology (Fig. 3g).

Finally, we checked the expression of the TIC surface marker $\alpha 2 \delta 1$ after knockdown of the four miRNAs. As shown in Fig. 3h, clear subpopulations of $\alpha 2 \delta 1^{+}$cells emerged on individual knockdown of the four miRNAs in $\alpha 2 \delta 1^{-}$ Huh7 cells. Furthermore, western blot analyses demonstrated that the expression of $\alpha 2 \delta 1$ was also upregulated after knockdown of each of the four miRNAs (Fig. 3i). On the contrary, the expression of $\alpha 2 \delta 1$ decreased significantly in Hep-12 cells on the switch of let-7c, miR-200b, miR-222 and miR-424 expression (Fig. 3j).

Therefore, knockdown the four miRNAs individually was sufficient to convert $\alpha 2 \delta 1^{-}$cells into TICs with stem cell-like properties.

$P B X 3$ is a bonafide common target of the four miRNAs. Given that all the four miRNAs can suppress the properties of $\alpha 2 \delta 1^{+}$ HCC TICs, we hypothesized that the common genes directly targeted by them may play central roles in the determination of HCC TIC properties. Indeed, seven genes, including $A D R B K 2$, HIPK2, PBX3, PLEKHA6, PRKAB2, RIMS3 and TAF9B, were found to be potential common targets of the four miRNAs (Fig. 4a) by merging the list of upregulated genes in the $\alpha 2 \delta 1^{+}$ TICs obtained by Affymetrix microarray mRNA hybridization (our unpublished data) with predicted target genes of each of the four miRNAs using the miRWalk algorithm ${ }^{33}$.

$P B X 3$ belongs to the highly conserved PBX (Pre-B-cell leukaemia homeobox) group of proteins, which in turn belongs to the PBC family of TALE (three-amino-acid loop extension) superclass of homeodomain proteins. It appears to be the most critical member of PBX family contributing to leukaemogenesis by serving as a cofactor of other homeodomain proteins such as HOX and MEIS ${ }^{34,35}$ to control gene expression, and has been identified as a direct target of let-7c, miR-181a/miR-181b, to function in colorectal cancer metastasis and leukaemogenesis, respectively ${ }^{36,37}$. Hence, $P B X 3$ was chosen to validate further whether it was directly targeted by the four miRNAs to regulate $\alpha 2 \delta 1^{+}$HCC TICs.

We first tested whether the expression of $P B X 3$ was negatively regulated by the four miRNAs. Compared with respective controls, the expression of $P B X 3$ decreased remarkably at both mRNA and protein levels when the four miRNAs were overexpressed individually in Hep-12 cells by lentivirus infection (Fig. 4b,c). Conversely, the expression of $P B X 3$ increased significantly after each of the four miRNAs was interfered in $\alpha 2 \delta 1^{-}$Huh7 cells at both mRNA and protein levels (Fig. 4d,e).

Rescue experiments were then performed to validate whether $P B X 3$ could functionally overcome the suppression effects of the four miRNAs on HCC TICs. Ectopic expression of $P B X 3$ in Hep12 cells individually overexpressed the four miRNAs resulted in tumorigenicity recovered when $10^{3}$ cells per site were transplanted into NOD/SCID mice (Fig. 4f), confirming that

Figure 4 | PBX3 is a common target of let-7c, miR-200b, miR-222 and $\mathbf{m i R - 4 2 4 . ~ ( a ) ~ V e n n ~ d i a g r a m ~ s h o w s ~ c a n d i d a t e ~ m R N A s ~ t h a t ~ w e r e ~ p r e d i c t e d ~ t o ~ b e ~}$ directly targeted by all the four miRNAs by in silico analysis of upregulated genes in the $\alpha 2 \delta 1^{+} \mathrm{HCC}$ TICs with predicted target genes of each of the four miRNAs using the miRWalk algorithm. (b,c) qRT-PCR and western blot assays were carried out to detect the expression of endogenous PBX3 at both mRNA (b) and protein (c) levels, respectively, in Hep-12 cells infected with lentiviruses harboring indicated miRNAs. (d,e) The expression of endogenous PBX3 at both mRNA (d) and protein (e) levels in FACS-sorted $\alpha 2 \delta 1^{-}$Huh7 cells after indicated miRNAs were knocked down by TuD RNAs. (f) The effects of $P B X 3$ on tumour formation of Hep-12 cells expressing indicated miRNAs. The numbers above each bar indicate the tumour-formation rates that are expressed as tumours formed/sites transplanted. The bars are means \pm s.d. of tumour weights. ${ }^{\star}$ Student's $t$-test. (g) Sequence alignment of the seed sequence of indicated miRNAs with portions of the PBX3 3'-UTR (UTR WT) as predicted using the TargetScan software. The sequences designed as 'Mut' are mutated sequences in the matched seed sites that were used for creating firefly luciferase reporter constructs. (h) Dual-luciferase reporter assay in Hep-12 cells transiently co-transfected with the indicated constructs. Data are the average \pm s.d. of three independent experiments. ${ }^{*}$ Student's $t$-test. (i-I) The inverse correlation of indicated miRNAs against PBX3 mRNA expression was determined across purified $\alpha 2 \delta 1^{+}$and $\alpha 2 \delta 1^{-}$subsets from indicated sources. Data represented fold difference of $\alpha 2 \delta 1^{+}$cells over their negative counterparts in the same categories. Error bars indicate s.d. of three independent experiments. ( $\mathbf{m}-\mathbf{p})$ The relationship between the expression of indicated miRNAs and PBX3 mRNA in clinical HCC tissues. 
downregulation of $P B X 3$ is necessary for the suppression roles of the four miRNAs.

Luciferase reporter assay was further carried out by employing a luciferase reporter vector containing the $3^{\prime}$-UTR of $P B X 3$ flanking all the putative binding sites of the four miRNAs. Mutations in the putative binding sites were created as controls (Fig. 4g). When each of the four miRNAs was co-transfected with the reporter vector, the luciferase activities of the wild-type (WT)

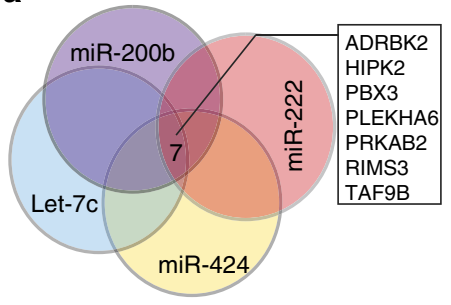

d

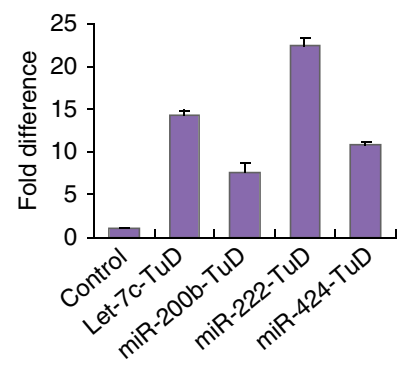

g

Mut $\quad 5$ '.. AAAACGAGgCUUUAUCGAAGAC UTR WT 5 '... AAAACACAGCUUUAUUACCUCA Let-7c 3' UUGGUAU'GUUUGGAUG--A'ÚG'GA'ǴU

Mut 5 '... UAGAGUGCUCACUUACGAACAU UTR WT $5^{\prime} \ldots$ UAGAGUGCUCACUUACUACCUC Let-7c $3^{\prime}$ UUGGUAUGUUGGAU'G'ÁU'ǴGA'G

Mut 5 '... GAUGGUCCCAGAUUUUGACGUC UTR WT 5 '... GAUGGUCCCAGAUUUCAGUAUU miR-200b 3' AGUAGUAAUGGUCC--G'Uं'ÁU'ÁA

Mut $\quad 5^{\prime}$...GAGUGUUACGUAUAG--CACGAUA UTR WT 5'.,GAGUGUUGUAUAUAG--UGUAGCA

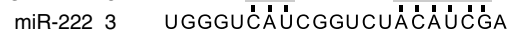

Mut 5 '.. AGACGAAGAU--ACUGUAGUAG UTR WT $5^{\prime} \ldots$ AGACGAAGAU--ACUUGCUGCU miR-424 3' AAGUUUUGUACUUA'A'C'GA'C'G'Á

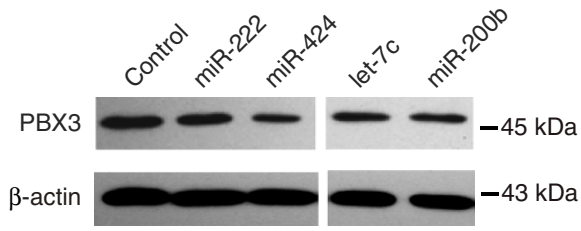

f

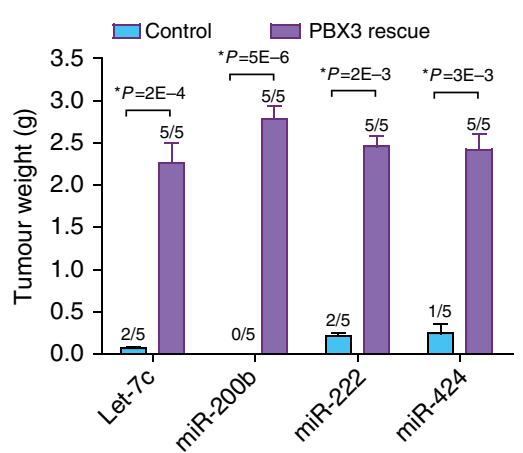

i

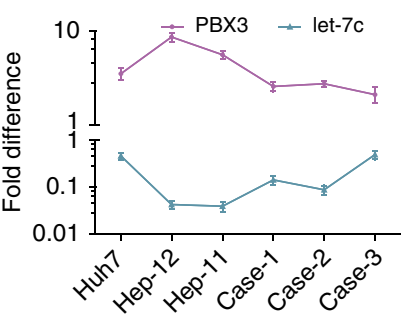

m

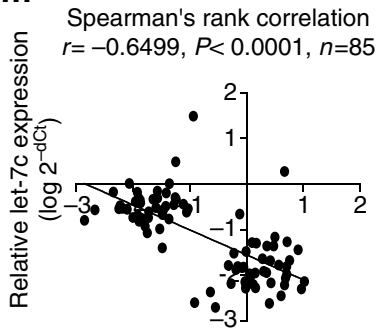

Relative PBX3 expression ( $\log 2^{-\mathrm{dCt}}$ )
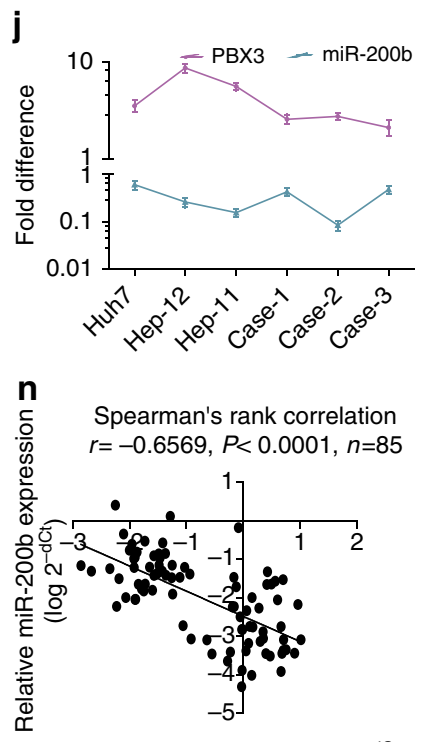

Relative PBX3 expression ( $\log 2^{-\mathrm{dCt}}$ ) k

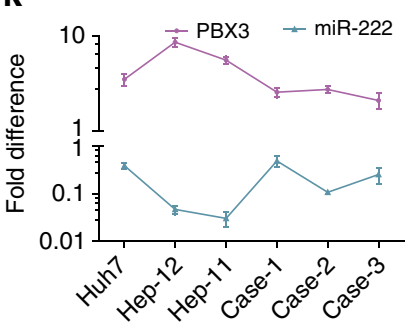

$$
\text { I }
$$

o

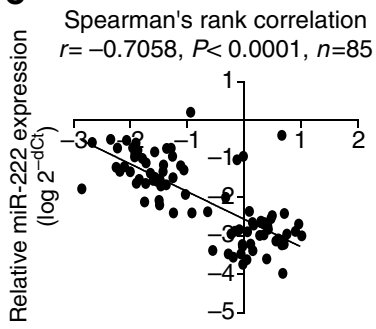

Relative PBX3 expression ( $\log 2^{-\mathrm{dCt}}$ )

I h
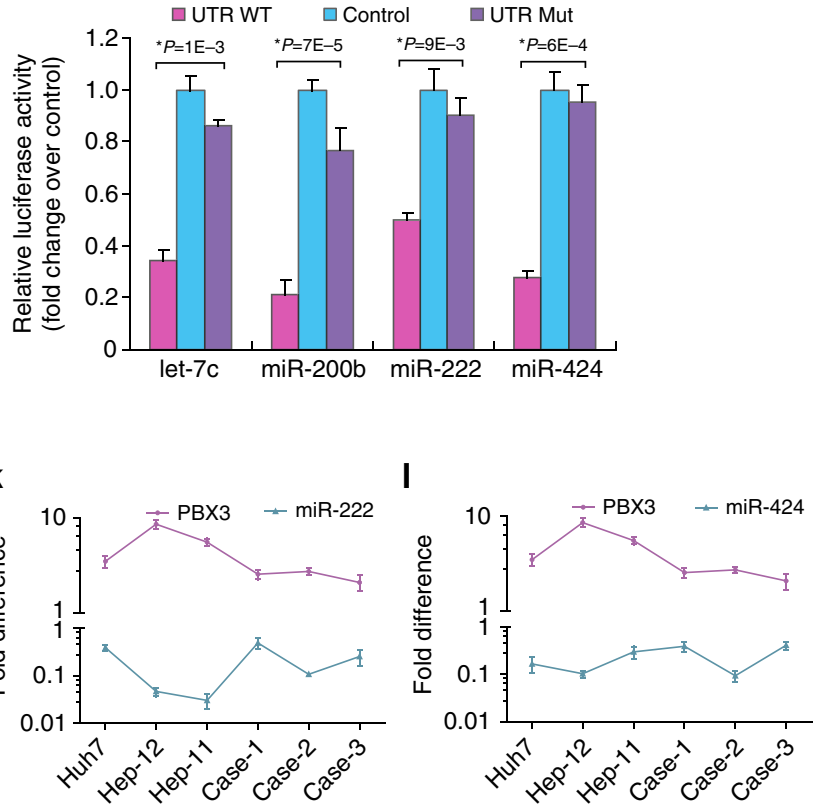

p

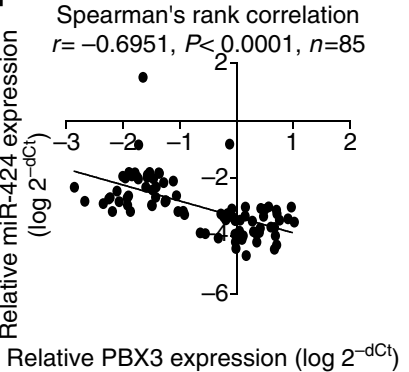


a

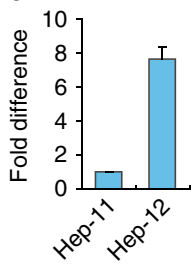

b

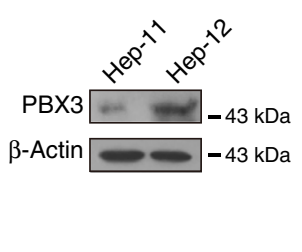

C

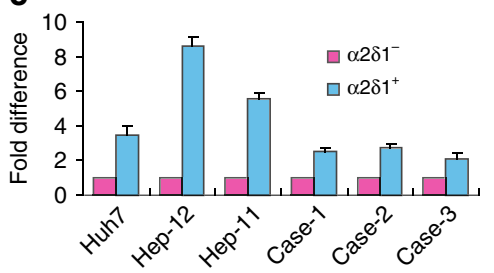

d

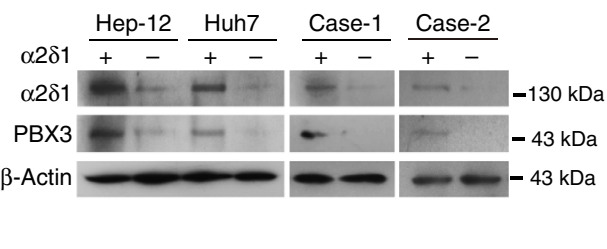

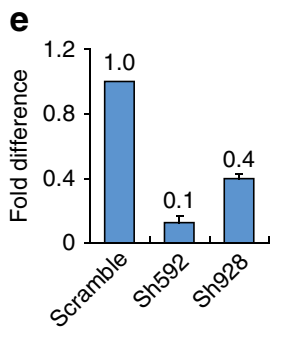

f
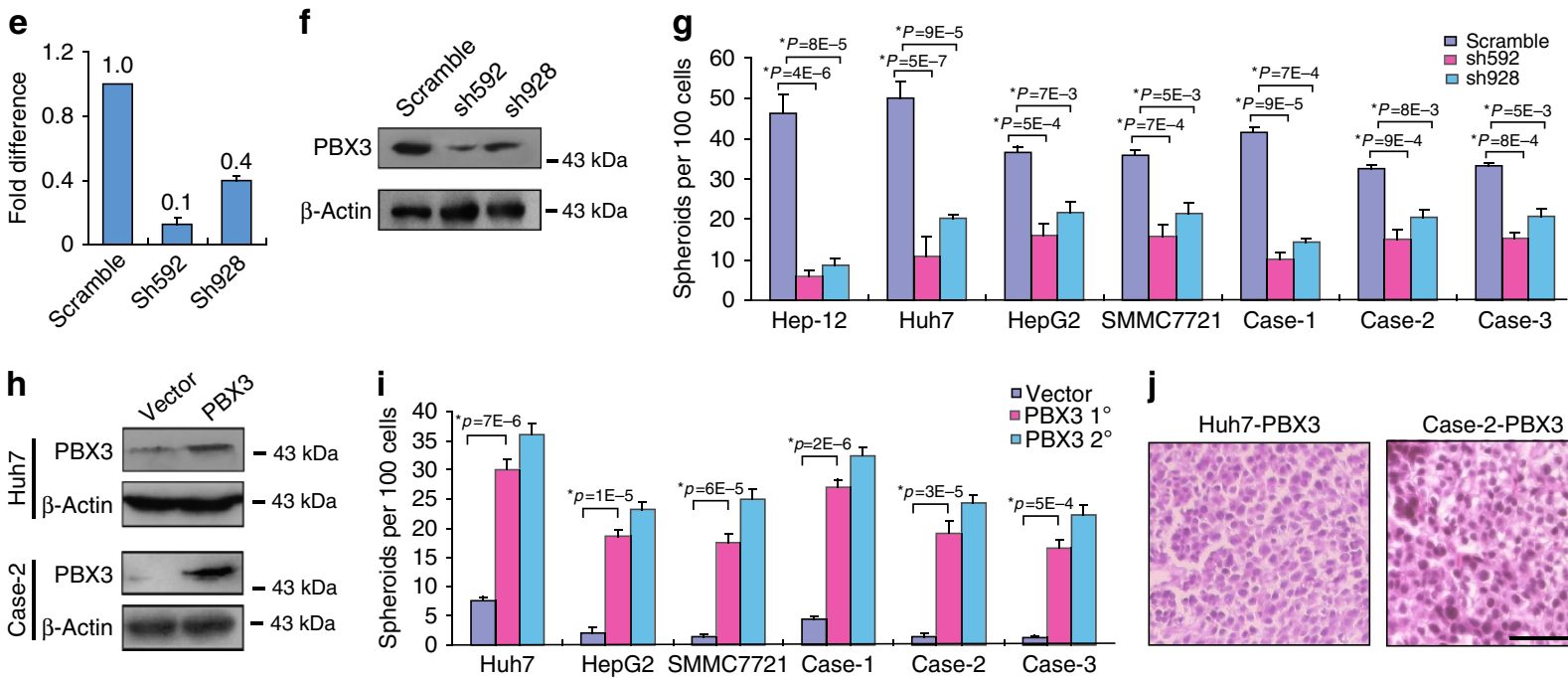

Case-2-PBX3

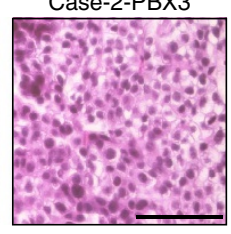

k
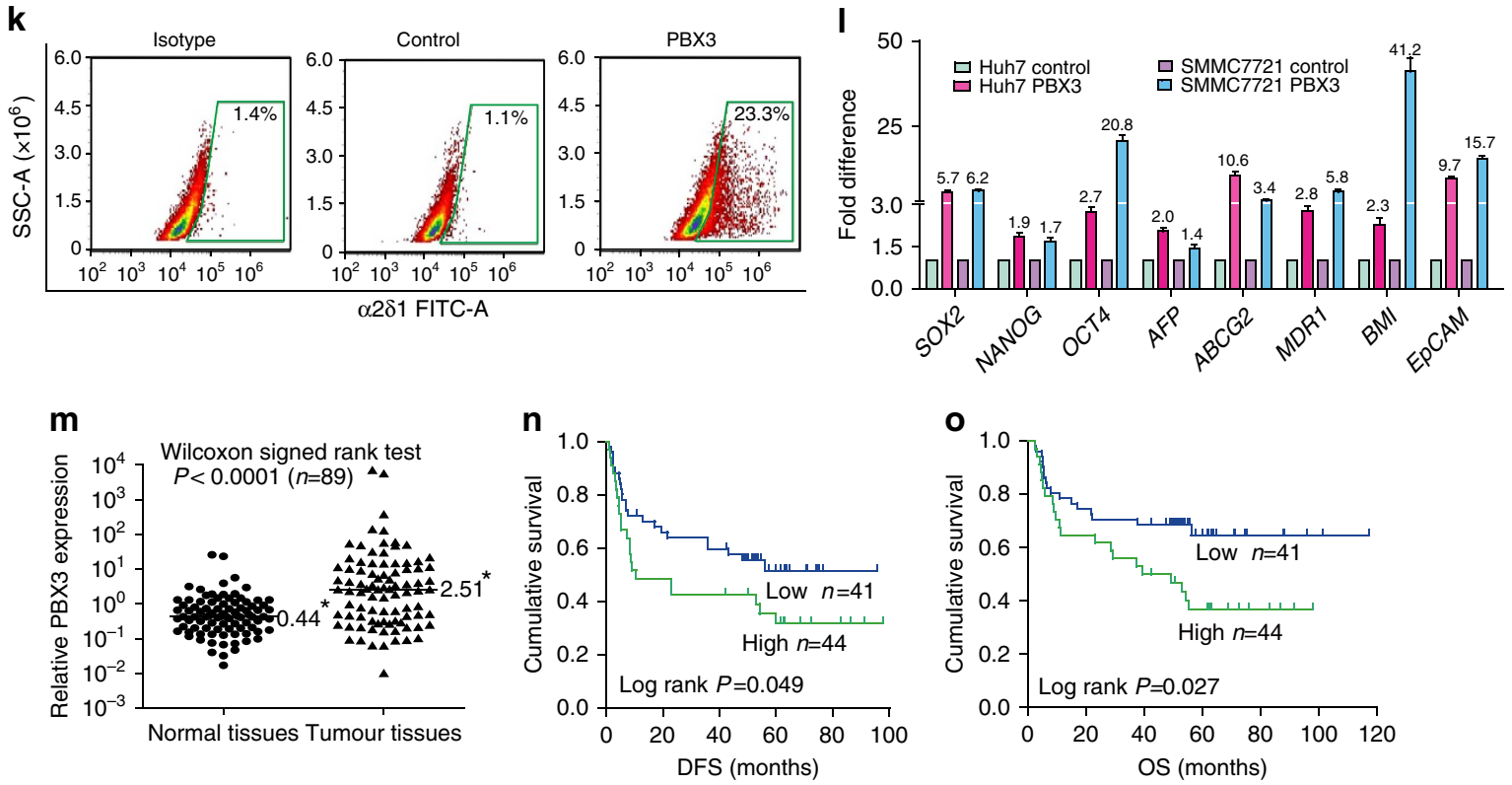

Figure 5 | PBX3 is a critical determinant of $\mathbf{\alpha 2 \delta} \mathbf{1}^{+}$HCC TICs. (a,b) The expression of PBX3 in Hep-11 and Hep-12 cells was detected at both mRNA and protein levels using qRT-PCR (a) and western blot (b), respectively. (c,d) The expression of PBX3 was compared between $\alpha 2 \delta 1^{+}$and $\alpha 2 \delta 1^{-}$fractions from indicated sources at both mRNA (c) and protein levels $(\mathbf{d}) .(\mathbf{e}, \mathbf{f})$ qRT-PCR $(\mathbf{e})$ and western blot (f) results showing the knockdown effects of PBX3 shRNAs on the expression of PBX3 in Hep- 12 cells at both mRNA and protein levels. $\beta$-actin serves as an internal control. (g) Sorted $\alpha 2 \delta 1^{+}$cells from indicated sources were infected with lentiviruses harbouring PBX3 shRNAs, followed by plating 100 cells per well into 96 -well plates to assay their sphere formation potential $(n=6)$. Data are the average \pm s.d. of three independent experiments. *Student's $t$-test. $(\mathbf{h}) \alpha 2 \delta 1^{-}$cells from indicated sources were infected with PBX3 lentivirus and assayed for PBX3 by western blot. (i) FACS-sorted $\alpha 2 \delta 1^{-}$cells from indicated sources were infected with PBX3 lentivirus and assayed for their sphere formation ability $(n=6)$. Data are the average \pm s.d. of three independent experiments. ${ }^{*}$ Student's $t$-test. (j) HE staining showing the histology of the tumours formed by $\alpha 2 \delta 1^{-}$cells from indicated sources after PBX3 lentivirus infection. Scale bar, $100 \mu \mathrm{m}$. (k) The percentage of $\alpha 2 \delta 1-$ positive cells were measured using flow cytometry in PBX3-lentivirus-infected Huh7 $\alpha 2 \delta 1^{-}$cells compared. with vector alone control. The data are representative of three experiments. (I) The expression of stem cell-related genes in $\alpha 2 \delta 1^{-}$cells overexpressing PBX3 was compared with vector alone controls using qRT-PCR. (m) The expression of PBX3 mRNA in HCC tissues was compared with adjacent normal tissues using qRT-PCR. ${ }^{\star}$ The numbers next to the horizontal lines are the median values $\left(2^{-\Delta C t}\right)$ for each group. $(\mathbf{n}, \mathbf{0})$ Kaplan-Meier curves for the DFS and OS of patients were compared between groups with high and low levels of PBX3 mRNA in HCC tissues, which were divided according to the cutoff of 2.51 , the median value of PBX3 relative to GAPDH mRNA. 


\begin{tabular}{|c|c|c|c|c|c|c|}
\hline \multirow[t]{2}{*}{ Cells } & \multicolumn{3}{|c|}{$\begin{array}{c}\text { PBX3 knocked down } \\
\alpha 2 \delta 1^{+} \text {cells }\end{array}$} & \multicolumn{3}{|c|}{$\begin{array}{c}\text { PBX3 overexpressed } \\
\alpha 2 \delta 1^{-} \text {cells }\end{array}$} \\
\hline & Group & $10^{3}$ & $10^{2}$ & Group & $10^{3}$ & $10^{2}$ \\
\hline \multirow[t]{3}{*}{ Hep-12 } & U6 & $5 / 5$ & $5 / 5$ & Control & $0 / 5$ & $0 / 3$ \\
\hline & Sh592 & $0 / 5$ & $0 / 5$ & $\mathrm{PBX} 3$ & $5 / 5$ & $3 / 3$ \\
\hline & Sh928 & $3 / 5$ & $1 / 5$ & ST & $5 / 5$ & ND \\
\hline \multirow[t]{3}{*}{ HepG2 } & U6 & $3 / 3$ & $3 / 3$ & Control & $0 / 3$ & $0 / 3$ \\
\hline & Sh592 & $0 / 3$ & $0 / 3$ & $\mathrm{~PB} \times 3$ & $3 / 3$ & $3 / 3$ \\
\hline & Sh928 & ND & ND & ST & $3 / 3$ & $3 / 3$ \\
\hline \multirow[t]{3}{*}{ SMMC7721 } & U6 & $3 / 3$ & $3 / 3$ & Control & $0 / 3$ & $1 / 3$ \\
\hline & Sh592 & $0 / 3$ & $0 / 3$ & PBX3 & $3 / 3$ & $3 / 3$ \\
\hline & Sh928 & ND & ND & ST & $3 / 3$ & $3 / 3$ \\
\hline \multirow[t]{3}{*}{ Case-1 } & U6 & $3 / 3$ & $3 / 3$ & Control & $0 / 5$ & $0 / 3$ \\
\hline & Sh592 & $0 / 3$ & $0 / 3$ & PBX3 & $4 / 5$ & $5 / 5$ \\
\hline & Sh928 & ND & ND & ST & $3 / 3$ & ND \\
\hline \multirow[t]{3}{*}{ Case-2 } & U6 & $3 / 3$ & $3 / 3$ & Control & $0 / 5$ & $0 / 3$ \\
\hline & Sh592 & $1 / 3$ & $0 / 3$ & PBX3 & $4 / 5$ & $3 / 3$ \\
\hline & Sh928 & ND & ND & ST & $5 / 5$ & $3 / 3$ \\
\hline \multirow[t]{3}{*}{ Case-3 } & U6 & $3 / 3$ & $3 / 3$ & Control & $0 / 3$ & $0 / 3$ \\
\hline & Sh592 & $0 / 3$ & $0 / 3$ & PBX3 & $3 / 3$ & $3 / 3$ \\
\hline & Sh928 & ND & ND & ST & $3 / 3$ & $3 / 3$ \\
\hline
\end{tabular}

3 -UTRs of $P B X 3$ were inhibited significantly compared with the respective mutant constructs (Fig. $4 \mathrm{~h}$ ).

Finally, the correlation between the expression of the four miRNAs and PBX3 mRNA was analysed in purified $\alpha 2 \delta 1^{+}$and $\alpha 2 \delta 1^{-}$fractions, as well as in clinical HCC samples. As expected, $P B X 3$ mRNA levels were negatively correlated with those of let$7 c$, miR-200b, miR-222 and miR-424 in purified $\alpha 2 \delta 1^{+}$and $\alpha 2 \delta 1^{-}$fractions across HCC cell lines and primary tissues (Fig. 4i-1), as well as in unsorted HCC samples (Fig. 4m-p).

These data indicate that the four miRNAs could suppress individually the properties of $\alpha 2 \delta 1^{+}$HCC TICs by directly targeting $P B X 3$.

$P B X 3$ determines the $\alpha 2 \delta 1^{+}$HCC TIC phenotypes. The above results prompted us to pursue further whether $P B X 3$ plays some roles in the acquisition and/or maintenance of HCC TIC properties. We first compared the expression of $P B X 3$ between Hep-12 and Hep-11 cell lines, and between the $\alpha 2 \delta 1^{+}$and $\alpha 2 \delta 1^{-}$ fractions sorted from HCC cell lines and primary HCC tissues. Indeed, the expression of $P B X 3$ was higher in Hep-12 cells than in Hep-11 cells, and was preferentially expressed in $\alpha 2 \delta 1^{+}$HCC TICs as compared with their negative counterparts at both mRNA and protein levels (Fig. 5a-d).

We then tested whether $P B X 3$ is required for the maintenance of TIC properties by knockdown of $P B X 3$ in $\alpha 2 \delta 1^{+}$TICs with short-hairpin RNA (shRNA). Stable knockdown $P B X 3$ in Hep-12 cells was achieved by infection with two lentiviruses harbouring $P B X 3$ shRNA sequence 592 and 928, respectively, with shRNA592 having a more prominent effect on $P B X 3$ silencing (Fig. 5e,f). Compared with scramble sequence, $P B X 3$ repression led to remarkable suppression of both the spheroid formation and the tumorigenic abilities of Hep-12 cells. Moreover, similar inhibition effects on the hepatosphere formation and the tumorigenicity were obtained when $\alpha 2 \delta 1^{+}$TICs sorted from HCC cell lines and primary tissues were infected with $P B X 3$ shRNA lentiviruses (Fig. 5g; Table 2), demonstrating that $P B X 3$ is necessary for the maintenance of $\alpha 2 \delta 1^{+}$TICs' self-renewal and tumorigenic capabilities.
In addition, ectopic expression of $P B X 3$ in $\alpha 2 \delta 1^{-}$fractions sorted from HCC cell lines and primary HCC was performed to address whether $P B X 3$ is sufficient to induce TIC-like phenotypes. Ectopic expression of $P B X 3$ in $\alpha 2 \delta 1^{-}$subsets (Fig. 5h) significantly enhanced the ability of these cells to initiate hepatosphere formation when grown in serum-free condition and to expand in subsequent serial propagations (Fig. 5i). Interestingly, these $\alpha 2 \delta 1^{-}$cells overexpressing $P B X 3$ exhibited an enhanced or newly acquired ability to initiate tumours when as few as 100 cells were injected s.c. in NOD/SCID mice compared with vector alone controls (Table 2). HE staining of xenografted tumours confirmed a primary HCC phenotype (Fig. 5j). Importantly, primary xenografted tumours formed by these $\alpha 2 \delta 1^{-}$cells overexpressing $P B X 3$ could also self-renew, as demonstrated by their ability to serially transplant in secondary NOD/SCID mouse recipients (Table 2). Finally, a distinct $\alpha 2 \delta 1^{+}$subpopulation was observed following forced expression of $P B X 3$ in $\alpha 2 \delta 1^{-}$Huh7 cells (Fig. 5k).

We finally address whether $P B X 3$ could regulate the genes associated with HCC TICs. The expression of a number of HCC TIC-associated genes such as NANOG, OCT4, ABCG2, MDR1 and $B M I 1$ was found to be remarkably increased following ectopic expression of $P B X 3$ in $\alpha 2 \delta 1^{-}$subpopulations, compared with vector controls (Fig. 5l).

Collectively, these data demonstrate that $P B X 3$ is sufficient to reprogramme $\alpha 2 \delta 1^{-}$HCC cells into TICs with stem cell-like properties and is necessary for the maintenance of $\alpha 2 \delta 1^{+}$TIC properties.

Clinical significance of $P B X 3$ in HCC patients. To determine the clinical significance of $P B X 3$ expression in HCC patients, we performed qRT-PCR analysis in 89 HCC patients. Compared with matched adjacent normal liver tissues, the expression of $P B X 3$ mRNA was significantly upregulated in HCC tissues (Fig. $5 \mathrm{~m}$ ). Further analysis of 85 HCC patients with detailed follow-up data indicated that the levels of $P B X 3$ mRNA in HCC tissues were positively correlated with the presence of hepatic cirrhosis, tumour size, rapid recurrence and short overall survival time (Supplementary Table 3). Kaplan-Meier curves show that those patients with higher levels of $P B X 3$ displayed short both disease-free and overall survival periods (Fig. 5n,o). Cox regression analysis identified the expression of $P B X 3$ mRNA in HCC as an independent risk factor of poor survival for patients (Supplementary Table 4).

Genome-wide identification of PBX3 target genes. To find the mechanisms underlying the roles of PBX3 in HCC TIC potential regulation genome widely, chromosome immunoprecipitationsequencing (ChIP-seq) using the specific rabbit anti-PBX3 polyclonal antibody and RNA-seq were performed in SMMC7721 cell line stably overexpressing $P B X 3$.

Model-based analysis of ChIP-Seq identified 39,508 PBX3bound regions, which were predominantly distributed in introns, intergenic regions and promoter within $3 \mathrm{~kb}$ of transcription start site (TSS) for a known gene (Fig. 6a). Furthermore, the PBX3binding peaks were enriched around the TSS (Fig. 6b). Association of PBX3-bound regions with annotated genes has identified 9,192 PBX3-bound nearest genes, reflecting an average number of 4.3 PBX3-bound regions per gene.

Compared with empty lentivirus-infected SMMC7721 cells, a total of 891 PBX3-responsive genes, including 479 upregulated (fold change $>2, P<0.01$ ) and 412 downregulated genes (fold change $<0.5, P<0.01$ ), was revealed in PBX3-overexpressed cells as detected using RNA-seq analysis (Fig. 6c; Supplementary Table 5). Database for Annotation, Visualization and Integrated 
a

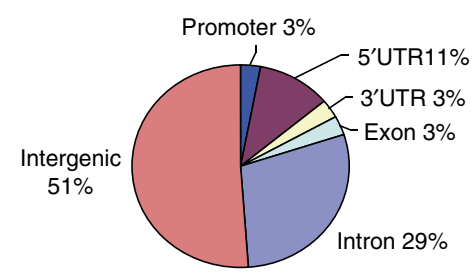

b

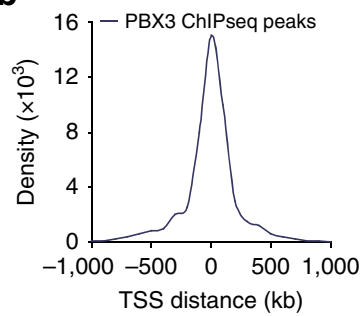

C

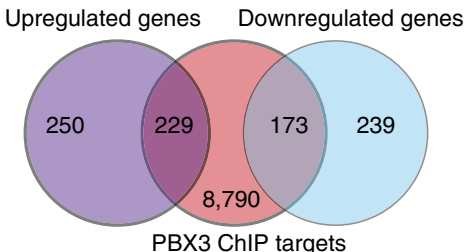

d
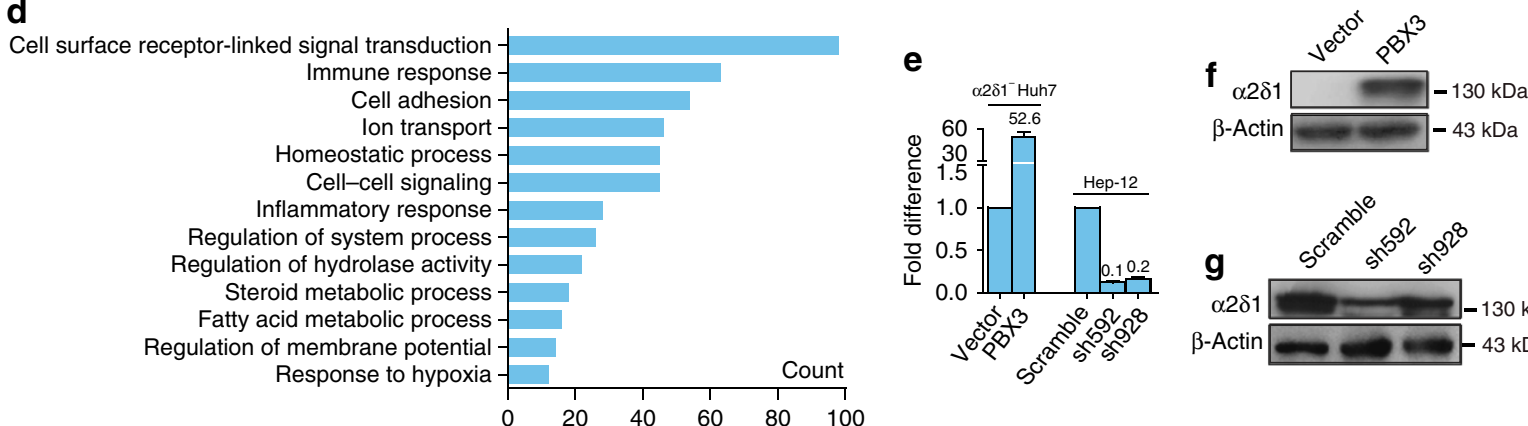

h

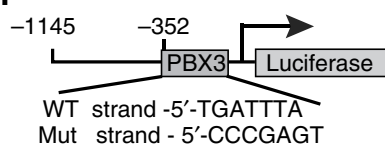

i

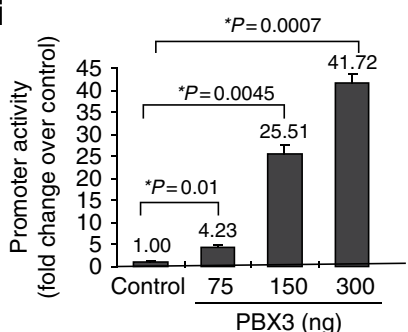

$\mathbf{k}$

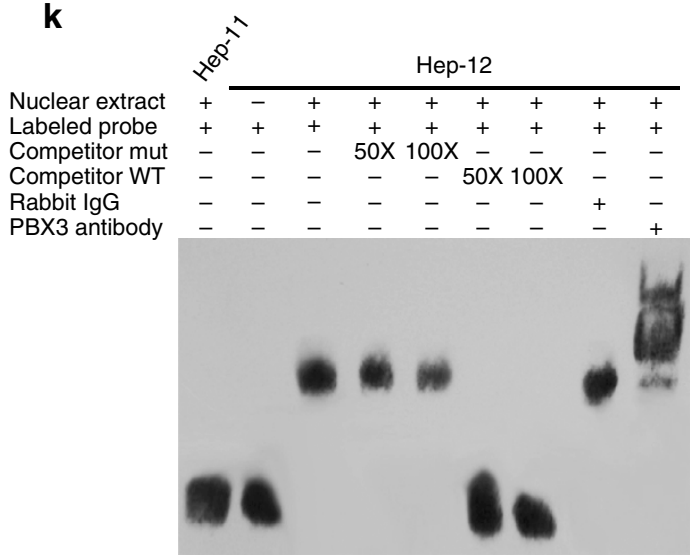

I

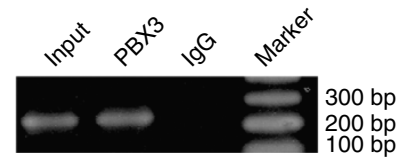

m

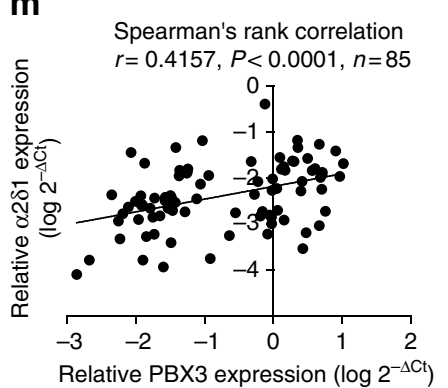

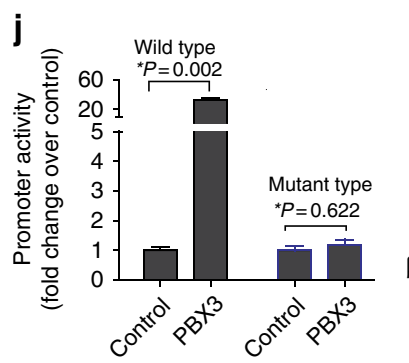

n

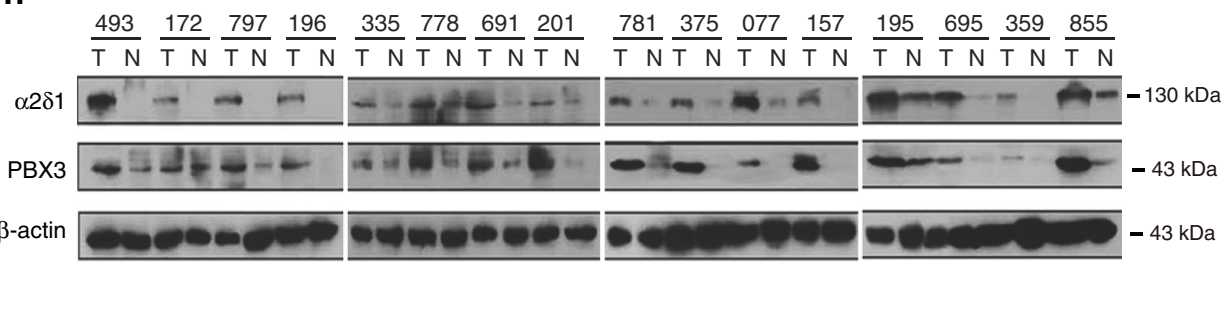

Figure 6 | The transcriptional programme driven by PBX3 in HCC TICs. (a) Piediagram summarizing the genomic occupancy of PBX3-bound regions as revealed by ChIP-seq. (b) Line diagram showing the distances of PBX3-bound peaks to the TSS. (c) Venn diagram illustrating direct target genes regulated by PBX3. (d) Gene Ontology (GO) term analysis of differentially regulated genes, as revealed using RNA-seq, in PBX3-overexpressed SMMC7721 cells over vector control. (e) qRT-PCR results showing $\alpha 2 \delta 1$ mRNA levels in PBX3-overexpressed $\alpha 2 \delta 1^{-}$Huh7 cells and PBX3 knockdown Hep-12 cells. (f,g) Western blot results demonstrating the change of endogenous $\alpha 2 \delta 1$ proteins following forced expression of $P B X 3$ in $\alpha 2 \delta 1^{-}$Huh7 cells or shRNA-mediated knockdown of $P B X 3$ in Hep-12 cells, respectively. (h) Schematic representation of the CACNA2D1 promoter that is fused to a luciferase reporter gene. The WT and mutant (Mut) sequences of the consensus potential PBX3-binding site used to make CACNA2D1 WT and Mut reporters, respectively, are indicated. (i,j) PBX3 activates the activity of the WT CACNA2D1 promoter in a dose-dependent manner (i), but not that of the mutant-type promoter containing mutant consensus potential PBX3-binding site (j). Luciferase reporter assay was performed in Hep-11 cells by co-transfection CACNA2D1 reporter with either pcDNA3-PBX3 or empty vector. Co-transfection of PRL-TK served as an internal control. Luciferase values were normalized to Renilla reporter activity. Data are presented as mean \pm s.d. of three independent experiments with triplicates. ${ }^{*}$ Student's t-test. (k) EMSA analysis was performed by incubating biotinlabeled oligonucleotide target containing consensus potential PBX3-binding site with Hep-11 or Hep-12 cell lysates. (I) ChIP assay was performed with control IgG or anti-PBX3 antibody in Hep-12 cells. The precipitated DNAs were amplified with primer pairs specific for flank sequence of the PBX3-binding sites in the CACNA2D1 promoter using PCR. (m) The correlation between the expression of PBX3 and its direct target CACNA2D1 in HCC tissues as detected using qRT-PCR. (n) Western blot results showing the correlation between PBX3 and $\alpha 2 \delta 1$ in 16 pairs of HCC (T) and adjacent normal tissues (N). 
Discovery (DAVID) gene ontology term analysis indicated that these genes were enriched for broad categories of biological processes (Fig. 6d; Supplementary Table 6). Notably, one of the significantly enriched gene groups was ion channels for calcium, potassium and sodium, as well as those involved in membrane potential regulation, suggesting that one of the critical mechanisms involved in PBX3-mediated TIC properties' regulation might involve these ions' influx/efflux. Importantly, functional annotation showed that the most significantly enriched genes (243 out of the 479 upregulated genes), including SOX2, SALL2, NOTCH3, WNT10A and LIN28A, were associated with embryo development (Supplementary Table 7). In addition, those genes associated with HCC TICs including the TIC markers CACNA2D1 (ref. 30), EpCAM (ref. 38) and THY1 (ref. 39), as well as multidrug-resistant gene $A B C B 1$ (MDR1), were also among the PBX3-upregulated genes.

Cross-analysis of PBX3-responsive genes with PBX3-motifbearing PBX3-bound genes has identified 229 upregulated genes and 173 downregulated genes, representing directly activated or repressed genes by PBX3, respectively (Fig. 6c; Supplementary Table 8). Many of the aforementioned genes such as CACNA2D1, EPCAM, SOX2, SALL2, NOTCH3 and WNT1OA are among PBX3 directly activated genes, indicating that $\mathrm{PBX} 3$ controls an essential transcriptional programme for HCC TICs.

Validation of the activation role of PBX3 on CACNA2D1. As a validation of the above RNA-seq and ChIP-seq results, we tested whether PBX3 directly activated the expression of CACNA2D1 in detail. We first detect whether PBX3 regulates the expression of endogenous $\alpha 2 \delta 1$. Forced expression of $P B X 3$ in $\alpha 2 \delta 1^{-}$Huh7 cells resulted in the activation of $\alpha 2 \delta 1$ expression at both mRNA and protein levels (Fig. 6e,f). On the other hand, repression of $P B X 3$ expression in $\alpha 2 \delta 1^{+}$Hep-12 cells led to the decrease in both $\alpha 2 \delta 1$ mRNA and protein levels (Fig. 6e,g).

We next constructed the luciferase reporter for the CACNA2D1 promoter (Fig. 6h) and performed luciferase reporter assay. As shown in Fig. 6i, a dose-dependent increase in the CACNA2D1 promoter-driven luciferase activity was observed in $P B X 3$ transiently transfected Hep-11 cells over vector control. When the PBX3 potential binding consensus site in the reporter vector was mutated, the activation effect of PBX3 was no longer detected (Fig. 6j), indicating that the binding site of PBX3 on CACNA2D1 promoter was responsible for the activation effect of PBX3.

We then carried out electrophoresis mobility shift assay using biotin-labeled probe containing the putative PBX3-binding site to test whether the potential PBX3-binding site does bind to PBX3. Incubation of the labeled WT PBX3-binding consensus oligonucleotide with the nuclear lysate of Hep-12 cells, which express high level of PBX3, clearly showed a retarded band compared with the labeled oligonucleotide without nuclear lysates or with nuclear lysates from Hep-11 cells expressing little PBX3. Furthermore, the retarded band was competed out specifically with an excess of unlabeled WT probe, and was supershifted by PBX3 antibody. In addition, the retarded band was not affected by competition with mutant PBX3-binding site oligonucleotide, or incubated with control IgG (Fig. 6k).

We also performed ChIP assay using Hep-12 cells to verify that PBX3 actually bound to the CACNA2D1 promoter in the native state. As shown in Fig. 6l, the promoter region flanking the putative PBX3-binding motif of CACNA2D1 was successfully precipitated by $\mathrm{PBX} 3$ antibody as evidenced by the presence of positive PCR band in the ChIP product.

As an additional supporting evidence that PBX3 activates the expression of CACNA2D1 in clinical HCC samples, the mRNA level of $P B X 3$ was found to be positively correlated with that of CACNA2D1 in 85 HCC tissues as detected using qRT-PCR (Fig. 6m). Western blot results in 16 pairs of tumour and paracancerous tissues from HCC patients also demonstrate a positive correlation between the expression of PBX3 and $\alpha 2 \delta 1$ at the protein level (Fig. 6n).

MiR-222 overcomes the effects of let-7c/miR-200b knockdown. Since the downregulation of let- $7 \mathrm{c}$ and $\mathrm{miR}-200 \mathrm{~b}$ and the upregulation of miR-222 were found extensively in HCC cells ${ }^{40-42}$, the fact that most HCC cells remain $\alpha 2 \delta 1$-negative led us to propose that miR-222 could rescue the effects of let-7c/miR-200b down-regulation on the expression of PBX3 and $\alpha 2 \delta 1$ in HCC cells. To test this hypothesis, we overexpressed miR-222 in Huh7 cells with let-7c and miR-200b knocked down simultaneously by respective Tud RNAs. While downregulation of let-7c and miR$200 \mathrm{~b}$ resulted in remarkable increase in PBX3 and $\alpha 2 \delta 1$ expression, further overexpression of miR-222 led to their expression decreased to the levels very close to those of parent Huh7 cells (Fig. 7a). Furthermore, the percentage of $\alpha 2 \delta 1^{+}$cells increased to $\sim 30.13 \%$ following let-7c and miR-200b knockdown, while it decreased to $5.27 \%$ when miR-222 was further overexpressed (Fig. 7b). These results indicate that it is the combinational effect of miR-222 upregulation and let-7c/miR$200 \mathrm{~b}$ downregulation at least that determines the expression status of $\alpha 2 \delta 1$ in most HCC cells.

Synergistic effects among the four miRNAs and their targets. To address whether any synergistic effects existed among the four miRNAs, we first overexpressed them simultaneously in the TIC-enriched Hep-12 cells (Supplementary Fig. 3A-D). The expression of PBX3 and $\alpha 2 \delta 1$ (Fig. 7c), as well as the spheroid formation efficiency (Fig. 7d) were suppressed at much stronger degrees in Hep-12 cells overexpressed the four miRNAs together than the four miRNAs individually. On the other hand, the expression of PBX3 and $\alpha 2 \delta 1$ (Fig. 7e), as well as the spheroid formation efficiency (Fig. 7f) increased much more significantly

Figure 7 | The combinational effects of the four miRNAs and their potential targets. (a) Western blot results showing the expression of PBX3 and $\alpha 2 \delta 1$ in Huh7 cells infected with indicated lentiviruses. (b) The percentage of $\alpha 2 \delta 1^{+}$cells was measured in Huh7 cells infected with indicated lentiviruses using flow cytometry. The data are representative of three experiments. (c) The expression of PBX 3 and $\alpha 2 \delta 1$ was assayed in $\alpha 2 \delta 1^{+}$Hep-12 cells overexpressing indicated miRNAs by western blots. (d) Hep-12 cells infected with lentiviruses harbouring indicated miRNAs were assayed for their spheroid formation ability $(n=6)$. Data are the average \pm s.d. of three independent experiments. ${ }^{\star} P \leq 0.05$ versus control; $\# P \leq 0.05$ versus any other group, Student's $t$-test. (e) Western blot results showing the expression of PBX3 and $\alpha 2 \delta 1$ in Huh7 cells following indicated miRNA knockdown. (f) The spheroid formation efficiency in Huh7 cells was measured following indicated miRNAs knockdown $(n=6)$. Data are the average \pm s.d. of three independent experiments. ${ }^{\star} P \leq 0.05$ versus control; $\# P \leq 0.05$ versus any other group, Student's $t$-test. (g-i) Western blot results showing the expression of indicated proteins in $\mathrm{HuH7}$ cells overexpressing indicated genes. $\beta$-actin serves as an internal control. (j) Representative photographs demonstrating the spheroids formed by Huh7 cells overexpressing ADRBK2, PBX3 or both. Scale bar, $250 \mu \mathrm{m}$; (k) Huh7 cells overexpressing the indicated genes were measured for their spheroid formation efficiency. Data are the average \pm s.d. of three independent experiments. $P$ values were calculated by Student's $t$-test. 
with the four miRNAs knocked down simultaneously in Huh7 cells, compared with individual knockdown of them (Supplementary Fig. 3E-H). These data indicate that synergistic effects do exist among the four miRNAs.

We then tested whether other common targets of the four miRNAs play any roles in TIC potential regulation, and whether there is any synergistic effect among them. We have selected three potential target genes, $A D R B K 2$ (also known as GRK3), PRKAB2 and HIPK2, which have been reportedly related to cancer ${ }^{43-45}$, for further study. Of the three genes, only $A D R B K 2$ was found to be able to induce Huh7 cells to acquire in vitro self-renewal ability as evidenced by increased spheroid formation efficiency, as well a

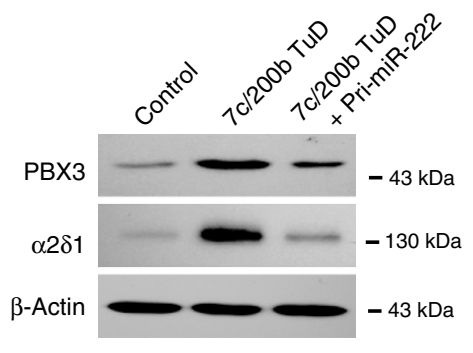

\section{b}

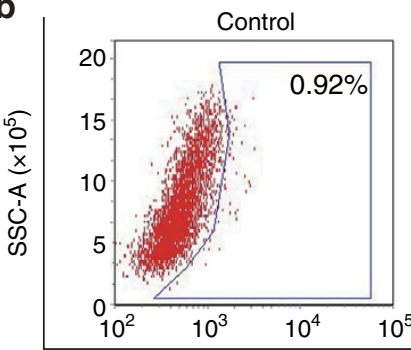

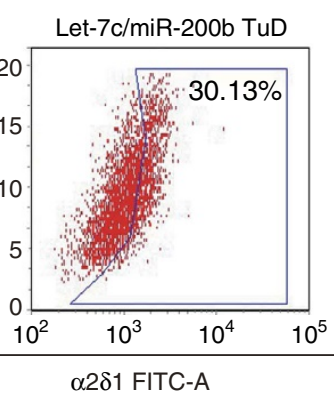

g
C

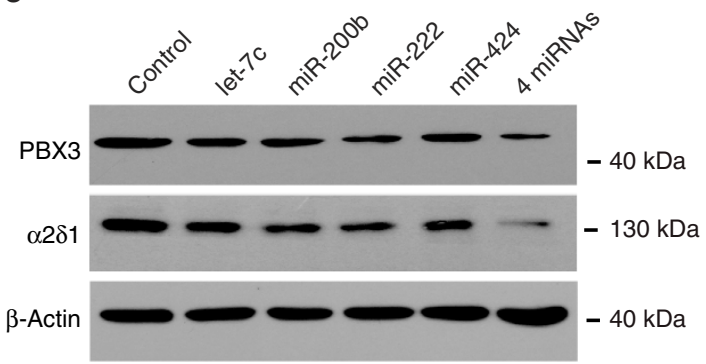

e

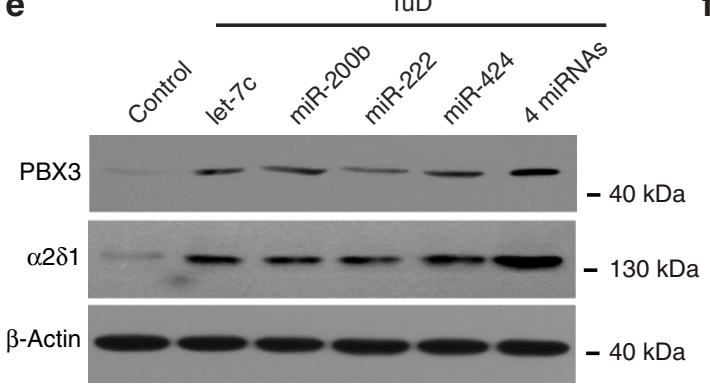

d

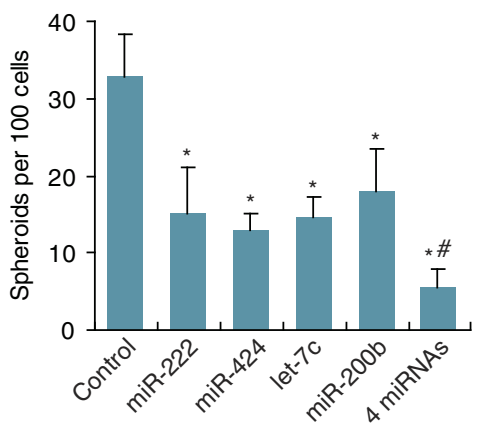

f

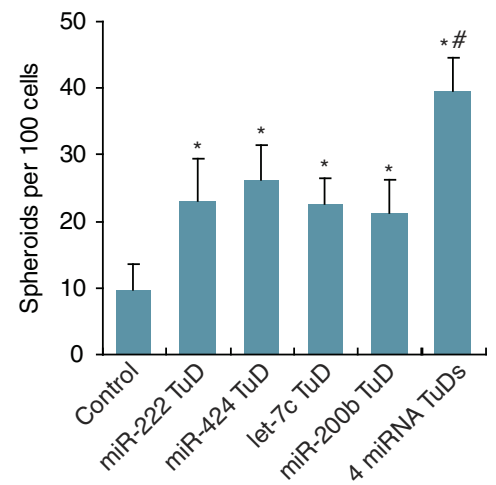

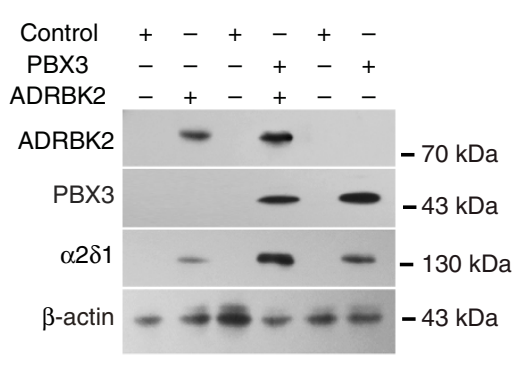

h

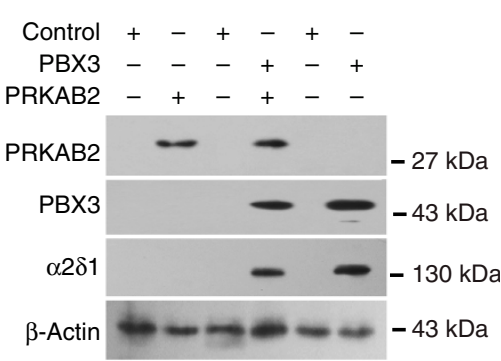

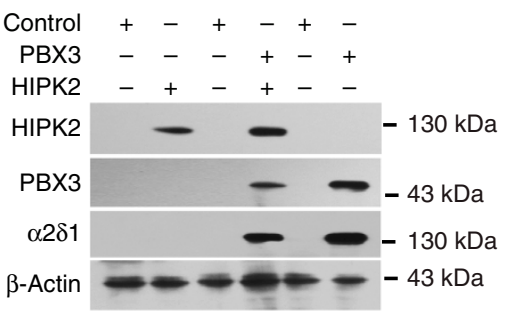
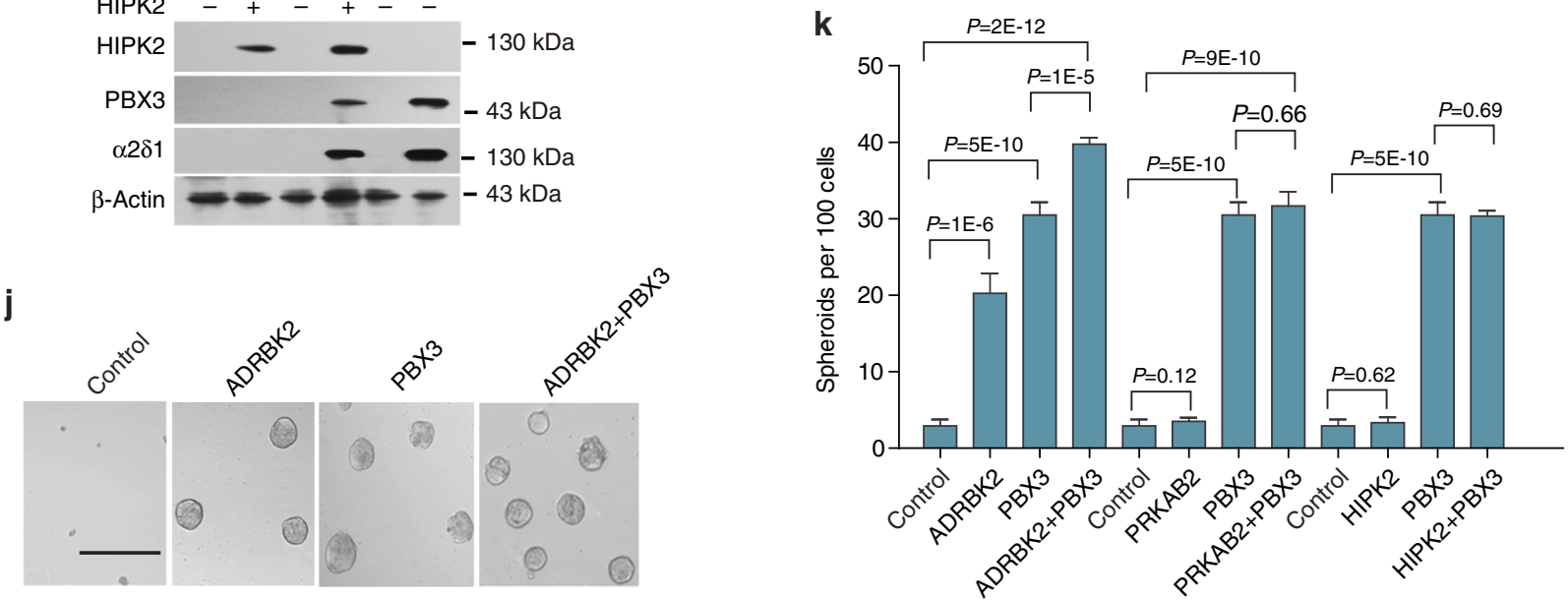


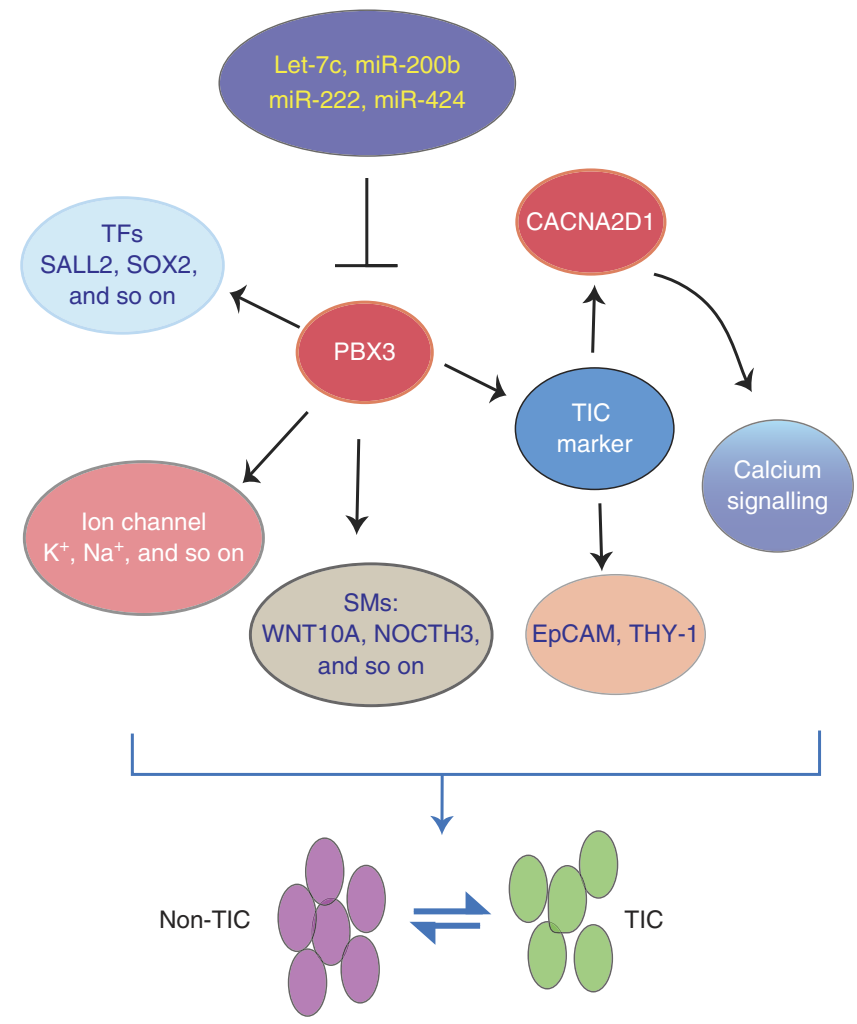

Figure 8 | A schematic illustration of the regulation pathway that determines the switch of HCC TICs and non-TICs. SM, signalling molecule; TF, transcriptional factor.

as to activate the expression of $\alpha 2 \delta 1$, although at a lesser degree compared with $P B X 3$. Furthermore, ADRBK2 could act synergistically with $P B X 3$, reprogramming Huh7 cells to acquire enhanced spheroid formation ability and elevated $\alpha 2 \delta 1$ expression (Fig. $7 \mathrm{~h}-\mathrm{k}$ ). The data indicate that at least two of the four miRNAs' common targets can work synergistically to determine the HCC TIC potential.

Taken together, all these results demonstrate that the downregulation of a set of miRNAs (let-7c, miR-200b, miR-222 and miR-424) has led to one of their direct targets, $P B X 3$, maintained above certain threshold, which in turn transcriptionally activates the expression of genes critical for stemness determination to maintain the HCC TIC phenotypes (Fig. 8).

\section{Discussion}

Functional screening genome widely of miRNAs involved in the regulation of TICs was hindered by limited number of TICs available from a given tumour, and by their unstable characteristics in regular culture condition. The fact that most of the recurrent HCC-originated Hep-12 cells remain as TICs even with prolonged in vitro culture has led to the identification of a TIC population expressing the $\alpha 2 \delta 1$ subunit $^{29,30}$. Here we developed a protocol by combining genome-wide miRNA profiling with softagar functional screening assay based on the Hep-12 cell line, to identify miRNAs that suppress the HCC TIC fate and have successfully identified a set of miRNAs, including four most effective miRNAs (let-7c, miR-200b, miR-222 and miR-424) that suppress the stemness and tumorigenicity of $\alpha 2 \delta 1^{+}$HCC TICs. This represents an efficiently functional screening strategy to identify all the possible miRNAs that control the properties of HCC TICs, and can be easily adopted to screen the key molecules involved in the regulation of HCC TIC properties.
Of the four most effective miRNAs identified, let-7c and miR$200 \mathrm{~b}$ belong to the let-7 and miRNA-200s families, respectively, of which the tumour-suppressor roles in TICs have been well appreciated $^{8,23,46}$, while miR-222 and miR-424 were first identified as TIC-suppressing miRNAs despite a tumoursuppressor role of both miRNAs has been identified in a number of cancers other than $\mathrm{HCC}^{47-50}$. Paradoxically, miR222 was previously regarded as oncogene in some reports because it could enhance some tumour cell growth by targeting CDK inhibitor p27 in vitro, and was found to be upregulated in HCC and some other types of cancers ${ }^{42,51,52}$; however, its tumourpromoting role was rarely validated in vivo. Our data support miR-222 to be a tumour suppressor in HCC by controlling the stemness and tumorigenicity of $\alpha 2 \delta 1^{+}$TICs, further confirming that the roles of miRNAs are cell context-dependent ${ }^{50}$. The study also indicates that a tumour suppressor on TICs was not necessarily downregulated in tumour tissues because the level reflects the status of the abundant non-TICs.

Most of the previous studies on miRNAs focused on single miRNA that targets one or more genes. Here we demonstrated that four downregulated miRNAs could target $P B X 3$ directly and synergistically at the transcriptional level to control the stemness and tumorigenicity of $\alpha 2 \delta 1^{+}$HCC TICs. Although knockdown of any of the four miRNAs could induce a TIC phenotype of $\alpha 2 \delta 1^{-}$cells, the facts that each of the four miRNAs alone is enough to suppress the TIC properties, and that those cells expressing decreased levels of let-7c and miR-200b, and elevated level of miR-222 remain to be non-TICs suggest that all of the miRNAs negatively regulate $P B X 3$ need to be downregulated to activate $\alpha 2 \delta 1^{+}$TICs. Furthermore, the four miRNAs could potentially regulate more common targets other than $P B X 3$; it is interesting to determine whether all these targets also contribute to the TIC potential determination, and if so, whether they work synergistically or antagonistically with PBX3. So far, ADRBK2, another common target of the four miRNAs, was found to work synergistically with PBX3 at least. Nevertheless, our current results indicate that the combination of multiple miRNAs that target the same gene should be considered when miRNAs involved in certain biological processes are investigated ${ }^{53}$.

PBX3 has been observed to be aberrantly overexpressed in a variety of tumours ${ }^{54-58}$; however, little is known about its biological roles in tumorigenesis and progression. Our current study demonstrates that $P B X 3$ plays determinant roles in HCC TIC properties, in particularly, controlling directly the expression of TIC surface markers $\alpha 2 \delta 1$ and EPCAM, stem cell master genes SOX2 and SALL2, as well as regulating the self-renewal and tumorigenicity potential. This observation is in consistent with the previous notion that the TALE homeodomain protein MEIS1 served a major role in establishing leukaemia stem cell potential and in determining leukaemia stem cell frequency probably by forming a complex with PBX proteins ${ }^{59}$. It would be interesting to further determine whether the roles of PBX3 in determining the TIC potential is also dependent on the interaction with other TALE homeodomain proteins such as MEIS and HOX family members.

In summary, we have identified four miRNAs that serve as negative regulators of $\alpha 2 \delta 1^{+}$HCC TICs by targeting directly $P B X 3$, which drives a stem cell-like transcriptional programme, including activation the genes related to calcium signalling, to enable HCC cells to reprogramme into TICs. Restoration of any of these miRNAs or interfering the expression/blocking the function of $\mathrm{PBX} 3$ in $\mathrm{HCC}$ cells may represent an promising strategy for future HCC treatment by reducing TICs. Future work is needed to address the driving events in the $\alpha 2 \delta 1^{+}$cell-induced HCC, especially how the four miRNAs are deregulated in the $\alpha 2 \delta 1^{+}$HCC TICs. 


\section{Methods}

Cell lines and clinical samples. HCC cell lines Hep-11 and Hep-12 were established by primary culture of a patient's primary and recurrent liver cancer tissues, respectively ${ }^{29}$. The Huh7, HepG2 and SMMC7721 cell lines were originated from Japan Society for the Promotion of Science (Tokyo, Japan), American Type Culture Collection (Manassas, VA) and the Second Military Medical University of China (Shanghai, China), respectively. All cell lines were cultivated in RPMI 1640 medium supplemented with $10 \%$ fetal bovine serum, $100 \mathrm{U} \mathrm{ml}^{-1}$ penicillin and $100 \mathrm{mg} \mathrm{ml}^{-1}$ streptomycin (Invitrogen, Grand Island, NY, USA) in a humidified atmosphere of $5 \% \mathrm{CO}_{2}$ at $37^{\circ} \mathrm{C}$. They were authenticated using polymorphic short tandem repeat loci and were tested for no mycoplasma contamination before the study initiated. Primary HCC specimens and matched adjacent normal tissues were collected and snap-frozen into liquid nitrogen from patients at the time of hepatectomy performed at the Beijing Cancer Hospital with written informed consent. Some primary HCC tissues were minced and dissociated into single cells by collagenase IV digestion for TIC isolation, or xenografted into NOD/SCID mice for repeated use. Acquisition and use of these tissues were approved by the Ethics Committee of Peking University Cancer Hospital.

Immunofluorescent staining and flow cytometry analysis. Cells were detached from culture by $0.02 \%$ EDTA/PBS or were dissociated from primary or xenografted tumours by mechanical homogenization, and were stained by rabbit anti- $\alpha 2 \delta 1$ polyclonal antibody (1:100 dilution, Sigma-Aldrich, St Louis, MO, "HPA008213), or Mab1B50-1 (ref. 30; $40 \mu \mathrm{g} \mu \mathrm{l}^{-1}$ ), which were pre-labeled using the LightningLink PE-Cy5 Labeling Kit (Innova Biosciences Ltd, Cambridge, UK) at $4{ }^{\circ} \mathrm{C}$ for $30 \mathrm{~min}$. Labeled samples were analysed or sorted on a FACSAria II flow cytometer (BD Biosciences, San Jose, CA).

Quantitative $\mu$ Paraflo miRNA microarray analysis. miRNA microarray assay was carried out using a service provider (LC Sciences, Houston, TX). The assay started from $5 \mu \mathrm{g}$ total RNA sample, which was size-fractionated using a YM-100 Microcon centrifugal filter (Millipore, Billerica, MA), and the small RNAs ( $<300$ nucleotides) isolated were $3^{\prime}$-extended with a poly(A) tail using poly(A) polymerase (NEB, Beverly, MA). An oligonucleotide tag was then ligated to the poly(A) tail for carrying out fluorescent dye staining later. Two different tags were used for the two RNA samples in dual-sample experiments. Hybridization was conducted overnight on a $\mu$ Paraflo microfluidic chip (miRHuman_13.0) using a microcirculation pump (Atactic Technologies, Houston, TX). On the microfluidic chip, each detection probe consisted of a chemically modified nucleotide-coding segment complementary to target miRNA (from miRNABase, http://microrna.sanger.ac.uk/sequences/) or other RNA (control sequences). Hybridization used $100 \mu \mathrm{l} 6 \times$ SSPE buffer $\left(0.90 \mathrm{M} \mathrm{NaCl}, 60 \mathrm{mmoll}^{-1} \mathrm{Na}_{2} \mathrm{HPO}_{4}, 6 \mathrm{mmoll}^{-1}\right.$ EDTA, pH 6.8) containing $25 \%$ formamide at $34^{\circ} \mathrm{C}$. After RNA hybridization, tagconjugating $\mathrm{Cy} 3$ and $\mathrm{Cy} 5$ dyes were circulated through the microfluidic chip for dye staining. Fluorescence images were collected using a laser scanner (GenePix 4000B, Molecular Devices, Sunnyvale, CA) and digitized using Array-Pro image analysis software (Media Cybernetics, Rockville, MD).

Spheroid formation assay. Spheroid formation was performed by plating 100 cells per well into 96-well ultra-low attachment plate (Corning Incorporated Life Sciences, Acton, MA) in serum-free DMEM/F12 medium (Invitrogen), supplemented with B27 (1:50; Invitrogen), $20 \mathrm{ng} \mathrm{ml}^{-1} \mathrm{bFGF}, 10 \mathrm{ng} \mathrm{ml}^{-1}$ HGF (Peprotech, Rocky Hill, NJ) and 1\% methylcellulose (Sigma-Aldrich). Cells were incubated in a $\mathrm{CO}_{2}$ incubator for 2-3 weeks and spheres were counted under a stereomicroscope (Olympus, Shinjuku-ku, Tokyo, Japan).

Tumorigenicity assay. For the assessment of tumour-formation abilities, cells were suspended in $50 \mu \mathrm{l}$ of plain RPMI1640 and Matrigel (BD Biosciences) mix (1:1) and transplanted s.c. into the armpit of 4- to 6-week-old NOD/SCID male and female mice (NOD.CB17-prkdscid/NcrCrl, Vitalriver, Beijing, China). Tumour formation was monitored weekly. All the animal protocols were performed under a Peking University Cancer Hospital Animal Care and Use Committee-approved protocol.

Total RNA extraction and qRT-PCR. Total RNAs were isolated with the miRNeasy Mini kit (Qiagen, Valencia, CA). For mature miRNA quantification, $50 \mathrm{ng}$ total RNA was polyadenylated by polyA polymerase (NEB), followed by reverse transcription with an oligo-dT adapter primer. For mRNA detection, cDNA was synthesized from total RNA using oligo $(\mathrm{dT})_{15}$ primers and Moloney murine leukaemia virus reverse transcriptase (Invitrogen, Carlsbad, CA). Real-time PCR was performed using SYBR Green PCR Master Mix (Toyobo, Osaka, Japan) on an ABI7500 PCR machine. Results were normalized to U6 for miRNA detection and GAPDH for mRNA measurement. Fold change was calculated by the $2^{-\Delta \Delta C t}$ method where $\Delta C_{\mathrm{t}}=C_{\mathrm{t}(\mathrm{Target})}-C_{\mathrm{t}(\text { Reference) }}$. All the primers were listed in Supplementary Table 9 . The specificity of the PCR was confirmed by melting curves and PCR product sequencing.
Soft-agar assay. Approximately 1,000 cells of each miRNA-transfected cell pool were plated in $0.5 \mathrm{ml}$ of $0.3 \%$ (w/v) Noble Agar (Difco, Detroit, MI) in culture medium on a solidified basal layer agar $(0.5 \mathrm{ml}$ of $0.5 \%$ agar in medium) per well in 24 -well culture plates, 6 wells per group. At 2 weeks after seeding, the colonies that contain at least 50 cells were counted under a microscope.

Fluorescence in situ hybridization of miRNAs. In situ hybridization of miRNAs was performed according to the miRCURY LNA microRNA ISH Protocol by using specific miRNA probes labeled with Digoxin (Exiqon, Vedbaek, Denmark). Images were captured on a Leica SP5 confocal microscope (Leica, Wetzlar, Germany).

Vector construction. All the constructs were made by standard DNA recombination techniques. The human pri-miRNA sequences containing each pre-miRNA and flanking sequence on both sides of pre-miRNA were amplified using PCR from genomic DNA using primers listed in Supplementary Table 9, and were subsequently cloned into pcDNA3.0, and/or lentiviral shuttle vector plenti6 (Invitrogen), or tetracycline-regulated plenti6-TREpitt vector, which was constructed in our previous study. The construction of PBX3 expression vector was described in our earlier paper ${ }^{36}$

For knockdown of miRNA expression, TuD RNAs against let-7c (5'-GGAT CCgacggcgctaggatcatcaacaaccatacaaccatcttactacctcacaagtattctggtcacagaatacaacaacca tacaaccatcttactacctcacaagatgatcctagcgccgtctttttCTCGAG-3'), miR-200b (5'-GGA TCCgacggcgctaggatcatcaactcatcattaccaatctggcagtattacaagtattctggtcacagaatacaactcat cattaccaatctggcagtattacaagatgatcctagcgccgtctttttCTCGAG-3'), miR-222 (5'-GGA TCCgacggcg ctaggatcatcaacacccagtagccatctagatgtagctcaagtattctggtcacagaatacaacac ccagtagccatctagatgtagctcaagatgatcctagcgccgtctttttCTCGAG-3') and miR-424 (5'-GGATCCgacggcgctag gatcatcaacttcaaaacatgaatctattgctgctgcaagtattctggtcaca gaatacaacttcaaaacatgaatctattgctgctgcaagatgatcctagcgccgtctttttCTCGAG-3') were designed according to ref. 32. For PBX3 knockdown constructs, two shRNA sequences, including shRNA592 (5'-GAGATTGAAAGAATGGTGGGC-3') and shRNA928 (5'-AGACGGCCGTGACAGCTGCAC- $\left.3^{\prime}\right)$, were designed. These TuD RNAs and shRNAs were cloned into lentiviral shuttle vector plenti6-U6.

For miRNA sensor vectors, the $3^{\prime} \mathrm{UTR}$ s of $P B X 3$ containing WT or mutant mature miRNA complementary sequences were cloned into the luciferase reporter vector. For CACNA2D1 reporter vector, the putative promoter region containing potential PBX3-binding site was amplified using PCR and cloned into PGL3-basic vector (Promega Corporation, Madision, WI) fused to a luciferase reporter gene. For construction of the PBX3-binding site mutant CACNA2D1 promoter-driven reporter, site-directed mutagenesis was carried out to obtain the mutant sequence of the promoter, and was then subcloned into the same vector.

The PCR primers used for making these constructs are listed in Supplementary Table 9 . All the constructs were confirmed by sequencing.

Transfection of pcDNA3.0 constructs into Hep-12 cells was performed using Lipofectamine 2000 reagent (Invitrogen) and then stable cell lines were obtained by selection with $500 \mu \mathrm{g} \mathrm{ml}^{-1} \mathrm{G} 418$ (Invitrogen). Lentiviral constructs were transfected with the ViraPower Packaging Mix (Invitrogen) into 293FT cells to generate lentivirus. Cells infected with virus are selected by $5 \mu \mathrm{g} \mathrm{ml}^{-1}$ blasticidin (Invitrogen) and/or $500 \mu \mathrm{g} \mathrm{ml}^{-1} \mathrm{G} 418$. The pool of antibiotic-resistant cells were used for subsequent assay.

Western blot analysis. Cells were lysed in radioimmunoprecipitation assay buffer (Suolaibo Biotechnology Co. Ltd, Shanghai, China) containing complete protease and phosphatase inhibitor cocktail (Roche, Mannheim, Germany). Cell lysates were then resolved through 10\% SDS-PAGE, transferred on polyvinylidene difluoride membrane (Millipore) and probed by rabbit anti-PBX3 (1:3,000 dilution, Abcam, Cambridge, UK, ${ }^{\#}$ Ab109173), mouse anti- $\alpha 2 \delta 1$ (1:3,000 dilution, Sigma-Aldrich, ${ }^{\#}$ HPA008213) or mouse anti- $\beta$-actin (1:50,000 dilution, Roche, ${ }^{\# 1378996),}$ followed by incubation with horseradish peroxidase (HRP)-conjugated goat antirabbit(1:100,000 dilution, Jackson ImmunoResearch Laboratories, West Grove, PA, $\left.{ }^{\#} 111-035-003\right)$ or anti-mouse IgG (1:100,000 dilution, Jackson ImmunoResearch Laboratories, ${ }^{\# 115-035-003) ~ s e c o n d a r y ~ a n t i b o d y . ~ S i g n a l s ~ w e r e ~ d e t e c t e d ~ w i t h ~}$ Immobilon Western Chemiluminescent HRP substrate (Millipore). Images have been cropped for presentation. Full-size images are presented in Supplementary Figs $4-7$.

Luciferase reporter assay. To determine relative luciferase activity, cells were seeded into 24-well plates. Each reporter construct was co-transfected with pRL-TK plasmid expressing renilla luciferase, and pri-miRNA or PBX3 expression vector using lipofectamine 2000 (Invitrogen). After $24 \mathrm{~h}$, cell lysates were made using $1 \times$ passive lysis buffer (Promega Corporation) according to the manufacturer's instructions. Firefly and Renilla luciferase activities in cell lysates were measured by a FLUOstar Optima illuminometer (BMG Labtech, Offenburg, Germany) using the dual-luciferase reporter assay kit (Promega). Firefly luciferase activity was normalized to that of renilla luciferase for each sample.

Chromatin immunoprecipitation assay. The chromatin immunoprecipitation (ChIP) assay was performed with the ExactaChIP Kit according to the manufacturer's instruction (R\&D Systems, Minneapolis, MN). Briefly, Hep-12 cells or 
PBX3-transfected Huh7 cells were formaldehyde-crosslinked, and the DNA was sheared by sonication. The crosslinked whole-cell extract was used for immunoprecipitation with PBX3 antibody or control IgG. After overnight immunoprecipitation, each sample was washed with washing buffers. The DNA was eluted and purified using the PCR Purification Kit from Qiagen. PCR was performed with primers specific for human CACNA2D1 (forward: $5^{\prime}$-TTGCCTAAGAAGCCAG ACGG-3', reverse: $5^{\prime}$-AGGGCGACTTTGGAAACAGAC- $3^{\prime}$ ). The ChIP products were sequenced using Illumina Hi-Seq 2000 (Illumina Inc., San Diego, CA) by a service provider (BIOPICS, Peking University, Beijing, China).

ChIP-seq data analysis. For ChIP-seq analysis, Illumina sequencing short reads were aligned against the Human Reference Genome (assembly hg19) using the Burrows-Wheeler Aligner ${ }^{60}$ tool with default settings. Only unique non-duplicate reads were retained. Peaks, areas where there were significantly more enriched reads mapped in the ChIP sample than the input, were determined using MACS ${ }^{61}$ with the parameters of $P$ value 0.00001 and false discovery rate $5 \%$. The Bedtools software ${ }^{62}$ was used to determine the genes closest to PBX3-binding peaks and to calculate the distance between these peaks from TSS of their closest genes.

Electrophoretic mobility shift assay. Electrophoretic mobility shift assay (EMSA) was performed using the LightShift chemilluminescent EMSA kit (Pierce, Rockford, IL) essentially the same as described ${ }^{63}$. The double-stranded oligonucleotide used as a probe for EMSA corresponds to the following sequence in the putative PBX3 WT binding site of the CACNA2D1 promoter region, $5^{\prime}$-TCCTCCCTGAT TTATTCCCCCG- $3^{\prime}$. The consensus sequence TGATTTA was changed to TCGAGCG in the respective mutant PBX3-binding motif used in the competition experiments. Probes were end-labeled with biotin. Reaction mixture of $20 \mu \mathrm{l}$ containing binding buffer, $5 \%$ glycerol (v/v), $5 \mathrm{mM} \mathrm{MgCl}_{2}, 50 \mathrm{ng} \mathrm{ul}^{-1}$ Poly $(\mathrm{dI} \cdot \mathrm{dC})$, $0.05 \% \mathrm{NP}-40,150 \mathrm{mM} \mathrm{KCl}, 20 \mathrm{fmol}$ probe and $10 \mu \mathrm{g}$ nuclear extract, which was prepared using NE-PER Nuclear and Cytoplasmic Extraction (Pierce, Philadelphia, $\mathrm{PA}$ ), were incubated at room temperature for $30 \mathrm{~min}$. For competition assays, excess unlabeled double-strand nucleotides were added to the reaction mixture. Either control IgG or PBX3 polyclonal antibody was included in the reaction mixture for super-shift assay. The DNA-protein complexes were separated on $6 \%$ native polyacrylamide gel in $0.5 \times \mathrm{TBE}$ and were electrophoretically transferred on positively charged nylon membrane. DNA and membrane were crosslinked at $120 \mathrm{~mJ} \mathrm{~cm}^{-2}$ using ultraviolet crosslinker at $254 \mathrm{~nm}$. Signals were detected using the Chemiluminiscent Nucleic Acid Detection module (Pierce) according to the manufacturer's protocol.

RNA-seq data analysis. Total RNA was isolated from SMMC7721 cells infected with PBX3 expression and control vector lentiviruses. Samples were sequenced on an Illumina Hi-Seq 2000 with 100-bp paired end. FASTQ sequence reads were aligned to the human reference genome (hg19) with the Burrows-Wheeler AlignerBWA tool at default settings ${ }^{60}$. The expression level of each gene was expressed as number of reads per kilobase per million mapped reads calculated according to the formula published in ref. 64. Exact test based on a Poisson model was used for differential expression gene analysis. The fold change of the expression level of each gene was calculated as the ratio of PBX3 overexpression to vector alone control. Genes with $P<0.01$, fold change $>2$ were considered to be upregulated genes by PBX3. Genes with $P<0.01$, fold change value $<0.5$ were considered to be downregulated genes by PBX3. Gene ontology analysis of PBX3 target genes was performed using the NIH DAVID (http://david.abcc.ncifcrf.gov/).

Statistical analysis. All data were analysed with methods defined in the text using SPSS 13.0. A $P$ value $\leq 0.05$ was considered statistically significant.

\section{References}

1. Clarke, M. F. et al. Cancer stem cells--perspectives on current status and future directions: AACR Workshop on cancer stem cells. Cancer Res. 66, 9339-9344 (2006)

2. Clevers, H. The cancer stem cell: premises, promises and challenges. Nat. Med. 17, 313-319 (2011)

3. Visvader, J. E. \& Lindeman, G. J. Cancer stem cells: current status and evolving complexities. Cell Stem Cell 10, 717-728 (2012).

4. Frank, N. Y., Schatton, T. \& Frank, M. H. The therapeutic promise of the cancer stem cell concept. J. Clin. Invest. 120, 41-50 (2010).

5. De Vito, C. et al. A TARBP2-dependent miRNA expression profile underlies cancer stem cell properties and provides candidate therapeutic reagents in ewing sarcoma. Cancer Cell 21, 807-821 (2012).

6. Mani, S. A. et al. The epithelial-mesenchymal transition generates cells with properties of stem cells. Cell 133, 704-715 (2008).

7. Riggi, N. et al. EWS-FLI-1 modulates miRNA145 and SOX2 expression to initiate mesenchymal stem cell reprogramming toward Ewing sarcoma cancer stem cells. Genes Dev. 24, 916-932 (2010).

8. Shimono, Y. et al. Downregulation of miRNA-200c links breast cancer stem cells with normal stem cells. Cell 138, 592-603 (2009).
9. Liu, Y. et al. Mouse fibroblasts lacking RB1 function form spheres and undergo reprogramming to a cancer stem cell phenotype. Cell Stem Cell 4, 336-347 (2009).

10. He, G. et al. Identification of liver cancer progenitors whose malignant progression depends on autocrine IL-6 signaling. Cell 155, 384-396 (2013).

11. Chang, C.-J. et al. p53 regulates epithelial-mesenchymal transition and stem cell properties through modulating miRNAs. Nat. Cell Biol. 13, 317-U296 (2011).

12. Chaffer, C. L. et al. Poised chromatin at the ZEB1 promoter enables breast cancer cell plasticity and enhances tumorigenicity. Cell 154, 61-74 (2013).

13. Chaffer, C. L. et al. Normal and neoplastic nonstem cells can spontaneously convert to a stem-like state. Proc. Natl Acad. Sci. USA 108, 7950-7955 (2011).

14. Roesch, A. et al. A temporarily distinct subpopulation of slow-cycling melanoma cells is required for continuous tumor growth. Cell 141, 583-594 (2010).

15. Ivey, K. N. \& Srivastava, D. MicroRNAs as regulators of differentiation and cell fate decisions. Cell Stem Cell 7, 36-41 (2010).

16. Tay, Y., Zhang, J., Thomson, A. M., Lim, B. \& Rigoutsos, I. MicroRNAs to Nanog, Oct4 and Sox 2 coding regions modulate embryonic stem cell differentiation. Nature 455, 1124-1128 (2008).

17. Marson, A. et al. Connecting microRNA genes to the core transcriptional regulatory circuitry of embryonic stem cells. Cell 134, 521-533 (2008).

18. Wang, Y., Medvid, R., Melton, C., Jaenisch, R. \& Blelloch, R. DGCR8 is essential for microRNA biogenesis and silencing of embryonic stem cell selfrenewal. Nat. Genet. 39, 380-385 (2007).

19. Liu, C. \& Tang, D. G. MicroRNA regulation of cancer stem cells. Cancer Res. 71, 5950-5954 (2011).

20. DeSano, J. T. \& Xu, L. MicroRNA regulation of cancer stem cells and therapeutic implications. AAPS J. 11, 682-692 (2009).

21. Ma, S. et al. miR-130b Promotes CD133(+) liver tumor-initiating cell growth and self-renewal via tumor protein 53-induced nuclear protein 1. Cell Stem Cell 7, 694-707 (2010).

22. Ji, J. et al. Identification of microRNA-181 by genome-wide screening as a critical player in EpCAM-positive hepatic cancer stem cells. Hepatology 50, 472-480 (2009).

23. Yu, F. et al. let-7 regulates self renewal and tumorigenicity of breast cancer cells. Cell 131, 1109-1123 (2007).

24. Liu, C. et al. The microRNA miR-34a inhibits prostate cancer stem cells and metastasis by directly repressing CD44. Nat. Med. 17, 211-215 (2011).

25. Bu, P. et al. A microRNA miR-34a-regulated bimodal switch targets Notch in colon cancer stem cells. Cell Stem Cell 12, 602-615 (2013).

26. Yin, G. et al. TWISTing stemness, inflammation and proliferation of epithelial ovarian cancer cells through MIR199A2/214. Oncogene 29, 3545-3553 (2010).

27. Saini, S. et al. miRNA-708 control of CD44( + ) prostate cancer-initiating cells. Cancer Res. 72, 3618-3630 (2012).

28. Fareh, M. et al. The miR 302-367 cluster drastically affects self-renewal and infiltration properties of glioma-initiating cells through CXCR4 repression and consequent disruption of the SHH-GLI-NANOG network. Cell. Death Differ. 19, 232-244 (2012).

29. Xu, X. L. et al. The properties of tumor-initiating cells from a hepatocellular carcinoma patient's primary and recurrent tumor. Carcinogenesis 31, 167-174 (2010).

30. Zhao, W. et al. 1B50-1, a mAb raised against recurrent tumor cells, targets liver tumor-initiating cells by binding to the calcium channel $\alpha 2 \delta 1$ subunit. Cancer Cell 23, 541-556 (2013).

31. Sainz, B. \& Heeschen, C. Standing out from the crowd: cancer stem cells in hepatocellular carcinoma. Cancer Cell 23, 431-433 (2013).

32. Haraguchi, T., Ozaki, Y. \& Iba, H. Vectors expressing efficient RNA decoys achieve the long-term suppression of specific microRNA activity in mammalian cells. Nucl. Acid Res. 37, e43 (2009).

33. Dweep, H., Sticht, C., Pandey, P. \& Gretz, N. miRWalk-database: prediction of possible miRNA binding sites by 'walking' the genes of three genomes. J. Biomed. Inform. 44, 839-847 (2011).

34. Laurent, A., Bihan, R., Omilli, F., Deschamps, S. \& Pellerin, I. PBX proteins: much more than Hox cofactors. Int. J. Dev. Biol. 52, 9-20 (2008).

35. Li, Z. et al. PBX3 is an important cofactor of HOXA9 in leukemogenesis. Blood 121, 1422-1431 (2013)

36. Han, H. B. et al. Let-7c functions as a metastasis suppressor by targeting MMP11 and PBX3 in colorectal cancer. J. Pathol. 226, 544-555 (2012).

37. Li, Z. et al. Up-regulation of a HOXA-PBX3 homeobox-gene signature following down-regulation of miR-181 is associated with adverse prognosis in patients with cytogenetically abnormal AML. Blood 119, 2314-2324 (2012).

38. Yamashita, T. et al. EpCAM-positive hepatocellular carcinoma cells are tumor-initiating cells with stem/progenitor cell features. Gastroenterology 136, 1012-1024 (2009).

39. Yang, Z. F. et al. Significance of CD90 + cancer stem cells in human liver cancer. Cancer Cell 13, 153-166 (2008).

40. Hou, J. et al. Identification of miRNomes in human liver and hepatocellular carcinoma reveals miR-199a/b-3p as therapeutic target for hepatocellular carcinoma. Cancer Cell 19, 232-243 (2011). 
41. Dhayat, S. A. et al. The microRNA-200 family--a potential diagnostic marker in hepatocellular carcinoma? J. Surg. Oncol. 110, 430-438 (2014).

42. Wong, Q. W. et al. MiR-222 overexpression confers cell migratory advantages in hepatocellular carcinoma through enhancing AKT signaling. Clin. Cancer Res. 16, 867-875 (2010).

43. Li, W. et al. GRK3 is essential for metastatic cells and promotes prostate tumor progression. Proc. Natl Acad. Sci. USA 111, 1521-1526 (2014).

44. Lin, J. et al. Downregulation of HIPK2 increases resistance of bladder cancer cell to cisplatin by regulating Wip1. PLoS ONE 9, e98418 (2014).

45. Li, C., Liu, V. W., Chiu, P. M., Chan, D. W. \& Ngan, H. Y. Over-expressions of AMPK subunits in ovarian carcinomas with significant clinical implications. BMC Cancer 12, 357 (2012).

46. Liu, C. et al. Distinct microRNA expression profiles in prostate cancer stem/ progenitor cells and tumor-suppressive functions of let-7. Cancer Res. 72, 3393-3404 (2012).

47. $\mathrm{Xu}$, J. et al. Suppressed miR-424 expression via upregulation of target gene Chk1 contributes to the progression of cervical cancer. Oncogene 32, 976-987 (2013).

48. Pallasch, C. P. et al. miRNA deregulation by epigenetic silencing disrupts suppression of the oncogene PLAG1 in chronic lymphocytic leukemia. Blood 114, 3255-3264 (2009).

49. Izumiya, M., Okamoto, K., Tsuchiya, N. \& Nakagama, H. Functional screening using a microRNA virus library and microarrays: a new high-throughput assay to identify tumor-suppressive microRNAs. Carcinogenesis 31, 1354-1359 (2010).

50. Felli, N. et al. MicroRNAs 221 and 222 inhibit normal erythropoiesis and erythroleukemic cell growth via kit receptor down-modulation. Proc. Natl Acad. Sci. USA 102, 18081-18086 (2005).

51. le Sage, C. et al. Regulation of the p27Kip1 tumor suppressor by miR-221 and miR-222 promotes cancer cell proliferation. EMBO J. 26, 3699-3708 (2007).

52. Pineau, P. et al. miR-221 overexpression contributes to liver tumorigenesis. Proc. Natl Acad. Sci. USA 107, 264-269 (2010).

53. Peter, M. E. Targeting of mRNAs by multiple miRNAs: the next step. Oncogene 29, 2161-2164 (2010)

54. Li, Y. et al. PBX3 is overexpressed in gastric cancer and regulates cell proliferation. Tumour Biol. 35, 4363-4368 (2014).

55. Papagiannakopoulos, T. et al. Pro-neural miR-128 is a glioma tumor suppressor that targets mitogenic kinases. Oncogene 31, 1884-1895 (2012).

56. Zeisig, B. B. et al. Hoxa9 and Meis1 are key targets for MLL-ENL-mediated cellular immortalization. Mol. Cell Biol. 24, 617-628 (2004).

57. Lin, Y. W. \& Aplan, P. D. Gene expression profiling of precursor T-cell lymphoblastic leukemia/lymphoma identifies oncogenic pathways that are potential therapeutic targets. Leukemia 21, 1276-1284 (2007).

58. Novak, R. L. et al. Gene expression profiling and candidate gene resequencing identifies pathways and mutations important for malignant transformation caused by leukemogenic fusion genes. Exp. Hematol. 40, 1016-1027 (2012).
59. Wong, P., Iwasaki, M., Somervaille, T. C. P., So, C. W. E. \& Cleary, M. L. Meis is an essential and rate-limiting regulator of MLL leukemia stem cell potential. Genes Dev. 21, 2762-2774 (2007).

60. Li, H. \& Durbin, R. Fast and accurate short read alignment with BurrowsWheeler transform. Bioinformatics 25, 1754-1760 (2009).

61. Zhang, Y. et al. Model-based analysis of ChIP-Seq (MACS). Genome Biol. 9, R137 (2008).

62. Quinlan, A. R. \& Hall, I. M. BEDTools: a flexible suite of utilities for comparing genomic features. Bioinformatics 26, 841-842 (2010).

63. Lan, L. et al. Upregulation of myosin Va by Snail is involved in cancer cell migration and metastasis. Int. J. Cancer 126, 53-64 (2010).

64. Mortazavi, A., Williams, B. A., McCue, K., Schaeffer, L. \& Wold, B. Mapping and quantifying mammalian transcriptomes by RNA-Seq. Nat. Methods 5, 621-628 (2008).

\section{Acknowledgements}

This work was supported by NSFC-China (Grant Nos 81071733, 81330051 and 81201964), the "863" Project (Grant No. 2015AA020403), the '973' Program (No. 2010CB529402), Beijing Natural Science Foundation (No. 5122012) and Beijing Outstanding Talents Training Funds in Health Sciences (Grant No. 2011-2-24).

\section{Author contributions}

H.H. and Z.Z. designed and performed the experiments, analysed the data and wrote the manuscript; Y.D., W.Z., D.C. and S.L. performed the experiments and analysed the data J.Z. and J.L. assisted with ChIP-seq and RNA-seq data analyses; Z.S. assisted with miRNA FISH experiments; X.B. designed the experiments; B.X. performed clinical study.

\section{Additional information}

Accession codes. The miRNA microarray and PBX3 transcriptome (ChIP-seq and RNA-seq) data sets have been deposited in the Gene Expression Omnibus database with the accession IDs GSE71004 and GSE71262, respectively.

Supplementary Information accompanies this paper at http://www.nature.com/ naturecommunications

Competing financial interests: The authors declare no competing financial interests.

Reprints and permission information is available online at http://npg.nature.com/ reprintsandpermissions/

How to cite this article: Han, H. et al. PBX3 is targeted by multiple miRNAs and is essential for liver tumour-initiating cells. Nat. Commun. 6:8271 doi: $10.1038 /$ ncomms9271 (2015). 


\section{Author Correction: PBX3 is targeted by multiple miRNAs and is essential for liver tumour-initiating cells}

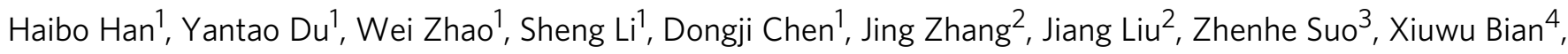
Baocai Xing ${ }^{5} \&$ Zhiqian Zhang ${ }^{1}$

Correction to: Nature Communications https://doi.org/10.1038/ncomms9271, published online 30 September 2015

This Article contains an error in Figure 3. In panel g, images representing miR-222-TuD and miR-424-TuD were both taken from the miR-222-TuD image. The correct version of Figure 3 is shown below as Fig. 1.

Published online: 21 May 2019

\footnotetext{
${ }^{1}$ Department of Cell Biology, Key Laboratory of Carcinogenesis and Translational Research (Ministry of Education/Beijing), Center for Molecular and Translational Medicine, Peking University Cancer Hospital and Institute, 52 Fucheng Road, Beijing 100142, China. ${ }^{2}$ Beijing Institute of Genomics, Chinese Academy of Sciences, Beijing 100101, China. ${ }^{3}$ Department of Pathology, Oslo University Hospital, Institute of Clinical Medicine, Faculty of Medicine, University of Oslo, Oslo 0379, Norway. ${ }^{4}$ Institute of Pathology and Southwest Cancer Center, Southwest Hospital, Third Military Medical University, Chongqing 400038, China. ${ }^{5}$ Department of Hepatobiliary Surgery I, Peking University Cancer Hospital and Institute, Beijing 100142, China. Correspondence and requests for materials should be addressed to Z.Z. (email: zlzqzhang@bjmu.edu.cn)
} 
a

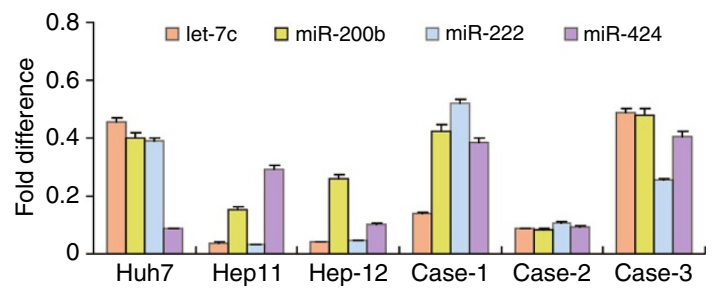

C

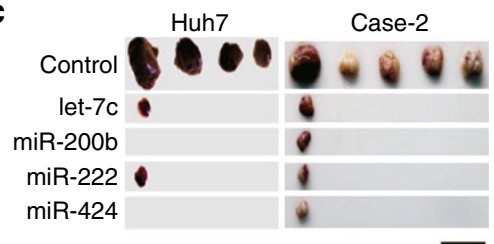

d

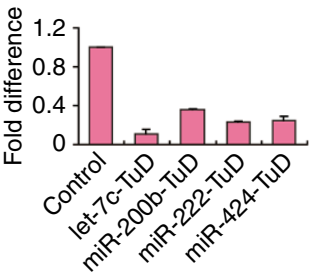

f

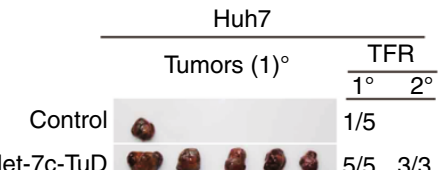

let-7c-TuD $5 / 5 \quad 3 / 3$ miR-200b-TuD 8000

miR-222-TuD $5 / 5 \quad 3 / 3$

miR-424-TuD $4 / 5 \quad 3 / 3$

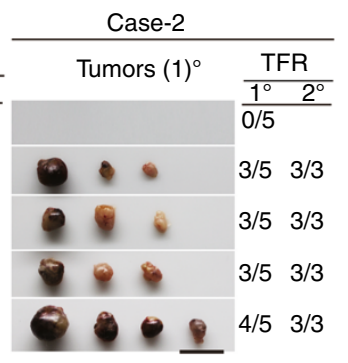

g

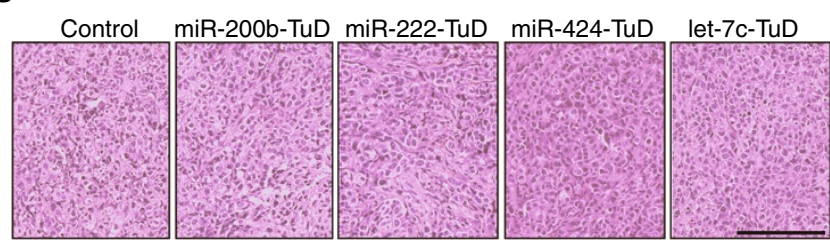

e
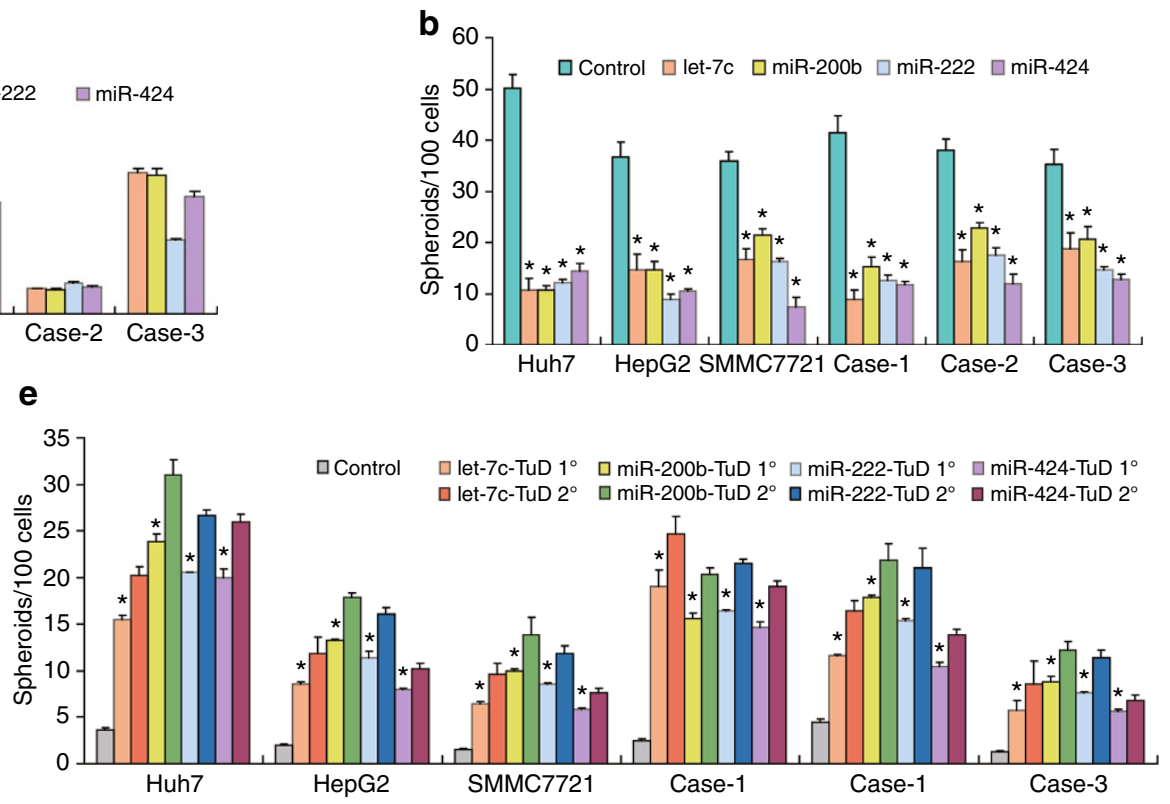

h
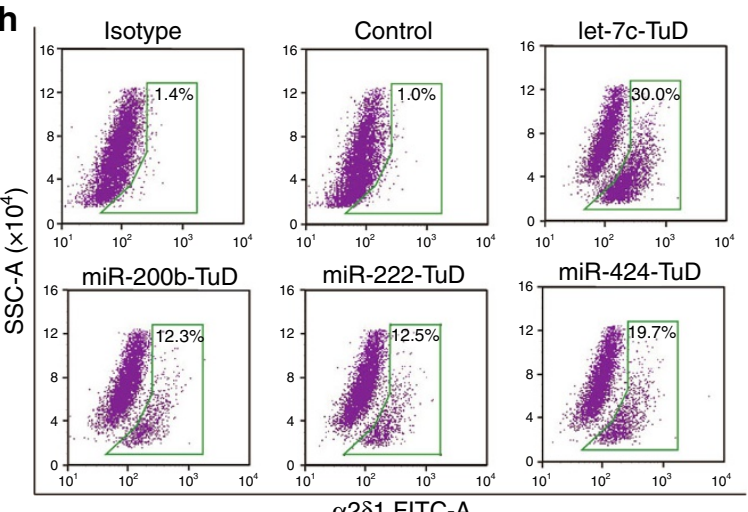

i

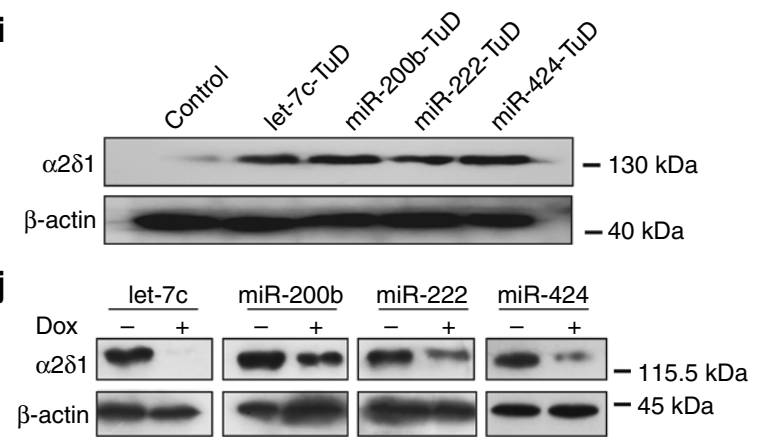

Fig. 1 